\title{
A convexity approach to dynamic output feedback robust MPC for LPV systems with bounded disturbances
}

\author{
Xubin Ping*, Sen Yang, Baocang Ding, Tarek Raïssi, and Zhiwu Li
}

\begin{abstract}
A convexity approach to dynamic output feedback robust model predictive control (OFRMPC) is proposed for linear parameter varying (LPV) systems with bounded disturbances. At each sampling time, the model parameters and disturbances are assumed to be unknown but bounded within pre-specified convex sets. Robust stability conditions on the augmented closed-loop system are derived using the techniques of robust positively invariant (RPI) set and the S-procedure. A convexity method reformulates the non-convex bilinear matrix inequalities (BMIs) problem as a convex optimization one such that the on-line computational burden is significantly reduced. The on-line optimized dynamic output feedback controller parameters steer the augmented states to converge within RPI sets and recursive feasibility of the optimization problem is guaranteed. Furthermore, bounds of the estimation error set are refreshed by updating the shape matrix of the future ellipsoidal estimation error set. The dynamic OFRMPC approach guarantees that the disturbance-free augmented closed-loop system (without consideration of disturbances) converges to the origin. In addition, when the system is subject to bounded disturbances, the augmented closed-loop system converges to a neighborhood of the origin. Two simulation examples are given to verify the effectiveness of the approach.
\end{abstract}

Keywords: Dynamic output feedback, model predictive control, linear parameter varying systems, uncertain systems.

\section{INTRODUCTION}

Robust model predictive control (RMPC) has been an active research topic since more than three decades [1-3]. This is due to the fact that RMPC is capable of dealing with multi-variable systems, and allowing for system uncertainties and physical constraints to be considered in control sequence optimization in a straightforward manner. The MPC feedback control sequence are computed based on receding horizon solving an open-loop optimal control problem subject to system uncertainties and physical constraints. The optimal control problem in MPC usually bases on system states. However, most of RMPC approaches are often formulated assuming that full system states are measurable. In many practical cases, full states are often unmeasurable, and only system outputs are available. This motivates extensive investigations of output feedback RMPC (OFRMPC) based on for example Luenberger state observer (e.g., [4-7, 10,11]) and dynamic output feedback controller (e.g., $[12,14-18]$ ). The interesting works on OFRMPC for constrained linear sys- tems with bounded disturbances can refer to [7-9], and to $[10-14,17]$ for systems that have both model parametric uncertainties represented by linear parameter varying (LPV) system with bounded disturbances.

Output feedback controller design is a theoretically challenging issue in control theory and has attracted considerable attention due to its great importance in practice. However, many of existing output feedback optimization problems often lead to bilinear matrix inequalities (BMIs) formulations. Nevertheless, optimization problems with BMIs constraints are intrinsically known to be non-convex and NP-hard in general $[19,20]$. Several methods of dealing with non-convex BMIs optimization in OFRMPC have been proposed. In $[4,17]$, the dynamic output feedback controller parameters take a parameter-dependent form such that the non-convex optimization problem is reformulated in terms of linear matrix inequalities (LMIs) and solved by convex optimization. In $[6,10,11]$, the state observer gain is off-line optimized, and the on-line controller design considers the dynamics of the estimation error determined by the off-line observer gain. Therefore,

Manuscript received xxxx, 2019; revised xxxx; accepted xxxx. Recommended by Associate Editor xxxx under the direction of Editor xxxx. This work was funded by the National Nature Science Foundation of China (61973243, 61403297), the Natural Science Basic Research Plan in Shaanxi Province of China (2017JQ6043).

Xubin Ping, Sen Yang, and Zhiwu Li are with the School of Electro-Mechanical Engineering, Xidian University, China (e-mails: xbping@xidian.edu.cn, yangsenxidian@163.com, and systemscontrol@gmail.com). Baocang Ding is with College of Automation, Chongqing University of Posts and Telecommunications, Chongqing 400065, China (e-mail: baocangding@126.com).. Tarek Raïssi is with CEDRIC-Lab, Conservatoire National des Arts et Métiers, Paris, France (e-mail: tarek.raissi@ cnam.fr). * Corresponding author. 
the difficulty in solving BMIs is avoided. Note that the optimization problems in $[4,6,10,11,17]$ are solved as the convex optimization, but the non-negative combining coefficients of LPV systems should be known in advance at each sampling time. However, when the non-negative combining coefficients of LPV systems are not exactly known (e.g., [12-16,18]), it is not trivial to solve the optimization problem by a convex optimization due to the existence of BMIs constraints. In [12-14], the cone complementary linearization (CCL) [21] approach is employed to deal with BMIs constraints, where a set of LMIs are iteratively solved to optimize a performance cost and dynamic output feedback controller parameters. Notice that on-line solving dynamic OFRMPC optimization via the CCL approach often suffers from large computational burden due to the iterative optimization. An off-line dynamic OFRMPC approach based on a look-up table method is considered in [14], where the computational burden is reduced but the control performance degrades compared with the on-line dynamic output feedback RMPC methods. This motives us to design an on-line convex dynamic OFRMPC optimization problem solved by a non-iterative optimization for LPV systems with unknown non-negative combining coefficients.

The main contribution of the paper is to present a convexity approach to dynamic OFRMPC for LPV systems with unknown non-negative combining coefficients and bounded disturbances. By employing robust positively invariant (RPI) sets and the S-procedure [25], an optimization problem with the update of the ellipsoidal estimation error set is formulated to ensure robust stability of the augmented closed-loop system. For the non-convex BMI conditions in the main optimization problem, the technique of Young's inequality is utilized to reformulate BMI conditions as LMIs, where some parameters are off-line searched to ensure the initial feasibility of the on-line main optimization problem. At each sampling time, the convex optimization problem is solved only one time to minimize the performance cost with respect to dynamic output feedback controller parameters. Compared with [12-14], the on-line main optimization is solved as a non-iterative, and therefore the on-line computational burden on the main optimization problem is significantly reduced. The recursive feasibility of the on-line optimization problem is guaranteed by ensuring that the augmented closed-loop system evolves within time-varying RPI sets. The on-line optimization problem guarantees that the disturbance-free augmented closed-loop system (without consideration of bounded disturbances) converges to the origin, and the augmented closed-loop system bounded within RPI sets is steered to a neighborhood of the origin when the system is subject to bounded disturbances.

Notations: Let $\mathbb{R}, \mathbb{R}_{+} \mathbb{Z}$ and $\mathbb{Z}_{+}$denote respectively the set of real numbers, non-negative real numbers, integer numbers, and non-negative integers. $\mathbb{Z}_{[s, k]}$ and $\mathbb{Z}_{[s, \infty)}$ denote the set of non-negative integers from $s$ to $k$, and the set of non-negative integers that are greater than or equal to $s$, where $s, k \in \mathbb{Z}_{+}$. For any vector $x$ and positivedefinite matrix $W,\|x\|_{W}^{2} \triangleq x^{\mathrm{T}} W x . x(i \mid k)$ is the value of $x$ at time $k+i$, predicted at time $k . x(0 \mid k)$ is the value of $x$ at time $k . \quad I$ is the identity matrix with appropriate dimensions. All vector inequalities are interpreted in an element-wise sense. An element belonging to $\operatorname{Co}\{\cdot\}$ means that it is a convex combination of the elements in $\{\cdot\} . P=\operatorname{diag}\left\{P_{1}, P_{2}\right\}$ denotes the diagonal matrix $P$ composed by the matrices $P_{1}$ and $P_{2}$. The symbol " $\star$ " induces a symmetric structure in matrices inequalities. A matrix or value with the superscript " $*$ " means that it is the optimal solution to an optimization problem. A matrix or value with the superscript "o" means that it is related with the final solution to the CCL approach. A matrix or value with the superscript " $t$ " means that it is the solution to an iterative optimization problem at the $t$-th optimization. The trace of the matrix $W$ is represented as $\operatorname{tr}\{W\}$. Denote $\mathcal{E}(p, M) \triangleq\left\{\xi \mid(\xi-p)^{\mathrm{T}} M(\xi-p) \leq 1\right\}$ as the ellipsoidal set associated with the center $p$, where $p, \xi \in \mathbb{R}^{n_{x}}$, and $M$ is a positive-definite matrix. When all the elements of the vector $p$ are $0, \mathcal{E}(p, M)$ is also denoted by $\mathcal{E}(M)$. The time-dependence of the MPC decision variable is often omitted for brevity.

\section{SYSTEM DESCRIPTION}

Consider the discrete-time uncertain LPV system

$$
\begin{aligned}
x(k+1) & =A(k) x(k)+B(k) u(k)+D(k) w(k), \\
y(k) & =C(k) x(k)+E(k) w(k),
\end{aligned}
$$

where $u \in \mathbb{R}^{n_{u}}, x \in \mathbb{R}^{n_{x}}, y \in \mathbb{R}^{n_{y}}$ and $w \in \mathbb{R}^{n_{w}}$ are respectively the system input, state, output and disturbance vectors. The disturbance $w(k) \in \mathcal{E}\left(P_{w}\right)$. The input and state are respectively bounded in the sets $\mathbb{U}$ and $\mathbb{S}$ given by

$$
\begin{aligned}
& \mathbb{U} \triangleq\{u(k) \mid-\bar{u} \leq u(k) \leq \bar{u}\}, \\
& \mathbb{S} \triangleq\{x(k+1) \mid-\bar{\psi} \leq x(k+1) \leq \bar{\psi}\},
\end{aligned}
$$

where $\bar{u}=\left[\bar{u}_{1}, \bar{u}_{2}, \cdots, \bar{u}_{n_{u}}\right]^{\mathrm{T}}, \bar{u}_{s}>0, s \in \mathbb{Z}_{\left[1, n_{u}\right.} ; \quad \bar{\psi}=$ $\left[\bar{\psi}_{1}, \bar{\psi}_{2}, \cdots, \bar{\psi}_{n_{x}}\right]^{\mathrm{T}}, \bar{\psi}_{f}>0, f \in \mathbb{Z}_{\left[1, n_{x}\right]}$. The system parameters $[A|B| C|D| E](k)$ are bounded within the convex set $\Omega \triangleq \operatorname{Co}\left\{\left[A_{l}\left|B_{l}\right| C_{l}\left|D_{l}\right| E_{l}\right]\right\}$. It means that there exist non-negative combining coefficients $\lambda_{l}(k), l \in$ $\mathbb{Z}_{[1, L]}$, such that $\sum_{l=1}^{L} \lambda_{l}(k)=1$ and $[A|B| C|D| E](k)=$ $\sum_{l=1}^{L} \lambda_{l}(k)\left[A_{l}\left|B_{l}\right| C_{l}\left|D_{l}\right| E_{l}\right]$, where $\left\{A_{l}\left|B_{l}\right| C_{l}\left|D_{l}\right| E_{l}\right\}$ are the sub-models of the LPV system. Furthermore, for the control problem to make sense, it is assumed that system (1) is controllable and observable for all admissible nonnegative combining coefficients of the LPV system.

For the controlled system (1), the dynamic output feedback controller [12-14] is given by

$$
\left\{\begin{aligned}
x_{c}(i+1 \mid k) & =A_{c}(k) x_{c}(i \mid k)+L_{c}(k) y(i \mid k), \\
u(i \mid k) & =F_{x}(k) x_{c}(i \mid k)+F_{y}(k) y(i \mid k), i \geq 0,
\end{aligned}\right.
$$


where $x_{c} \in \mathbb{R}^{n_{x}}$ is the controller state; $\left\{A_{c}, L_{c}\right\}$ are the controller gain matrices; $\left\{F_{x}, F_{y}\right\}$ are the feedback gain matrices. Denote the estimation error $e(k)=x(k)-x_{c}(k)$ and the augmented state $\tilde{x}(k)=\left[x_{c}^{\mathrm{T}}(k), e^{\mathrm{T}}(k)\right]^{\mathrm{T}}$. The augmented closed-loop system [14] based on (1) and (3) is

$$
\begin{aligned}
& \tilde{x}(i+1 \mid k)=T(k, i) \tilde{x}(i \mid k)+H(k, i) w(k+i), i \geq 0, \\
& \tilde{x}(k+1)=\tilde{x}(1 \mid k), \\
& {[T(k, i), H(k, i)]=\sum_{l=1}^{L} \sum_{j=1}^{L} \lambda_{l}(k+i) \lambda_{j}(k+i)\left[T_{l j}, H_{l j}\right],} \\
& T_{l j}=\left[\begin{array}{cc}
A_{c}(k)+L_{c}(k) C_{j} & L_{c}(k) C_{j} \\
\bar{\Delta}_{l j} & A_{l}+B_{l} F_{y}(k) C_{j}-L_{c}(k) C_{j}
\end{array}\right], \\
& \bar{\Delta}_{l j}=A_{l}+B_{l} F_{y}(k) C_{j}-L_{c}(k) C_{j}+B_{l} F_{x}(k)-A_{c}(k), \\
& H_{l j}=\left[\begin{array}{c}
L_{c}(k) E_{j} \\
B_{l} F_{y}(k) E_{j}+D_{l}-L_{c}(k) E_{j}
\end{array}\right] .
\end{aligned}
$$

In the following, it is assumed that the actual system state and bounded disturbances are unmeasurable, and the non-negative combining coefficients $\lambda_{l}(k), l \in \mathbb{Z}_{[1, L]}$, of the LPV system (1) are unknown at each sampling time. To stabilize the controlled system (1), the dynamic OFRMPC approach that optimizes the dynamic output feedback controller parameters in (3) by a convex optimization is developed to guarantee robust stability of the augmented closed-loop system (4).

\section{ROBUST STABILITY CONDITIONS AND PHYSICAL CONSTRAINTS}

In this Section, robust stability conditions on the augmented closed-loop system (4) are formulated in Theorem 1 according to the techniques of the S-procedure and RPI set. Then, the constraints on the input and state are considered in Lemma 2. To deal with non-convex BMIs in constraints, Lemma 1 is introduced.

Lemma 1: (Young's inequality [24]) Given two matrices $X$ and $Y$ with appropriate dimensions, then for any symmetric positive definite matrix $S$ and a scalar $\varepsilon>0$, we have

$$
\varepsilon X^{\mathrm{T}} S X+\frac{1}{\varepsilon} Y^{\mathrm{T}} S^{-1} Y \geq X^{\mathrm{T}} Y+Y^{\mathrm{T}} X .
$$

Remark 1: Suppose that $\Theta$ is a symmetric positive definite matrix. It can be seen that $\Theta+X^{\mathrm{T}} Y+Y^{\mathrm{T}} X \geq 0$ is equivalent to $\Theta-X^{\mathrm{T}}(-Y)-(-Y)^{\mathrm{T}} X \geq 0$. According to Lemma 1 and considering the quadratic form of matrix $Y(k),(6)$ is a sufficient condition for $\Theta-X^{\mathrm{T}}(-Y)-$ $(-Y)^{\mathrm{T}} X \geq 0$. Let $S=I$, and apply the Schur complement [22], (6) is equivalent to (7).

$$
\begin{aligned}
& \Theta-\varepsilon X^{\mathrm{T}} S X-\frac{1}{\varepsilon} Y^{\mathrm{T}} S^{-1} Y \geq 0, \\
& {\left[\begin{array}{ccc}
\Theta & \star & \star \\
X & \varepsilon^{-1} I & 0 \\
Y & 0 & \varepsilon I
\end{array}\right] \geq 0 . }
\end{aligned}
$$

\subsection{Robust stability conditions}

Theorem 1: Assume that at time $k \geq 0, e(k) \in$ $\mathcal{E}\left(Q_{e}^{-1}(k)\right)$, where $Q_{e}(k)$ is known at time $k$. The augmented state $\tilde{x}(k) \in \mathcal{E}\left(P^{-1}(k)\right)$ (the matrix $P(k) \triangleq$ $\operatorname{diag}\left\{P_{1}(k), P_{2}(k)\right\}$ and $\left.P^{-1}(k) \triangleq \operatorname{diag}\left\{M_{1}(k), M_{2}(k)\right\}\right)$ is satisfied if there exists a scalar $\rho(k) \in(0,1)$ such that (8) and (9) hold. For all possible $\tilde{x}(k) \in \mathcal{E}\left(P^{-1}(k)\right)$ and $w(k+i) \in \mathcal{E}\left(P_{w}\right), i \geq 0$, if there exist non-negative scalars $\left\{\alpha_{1}, \alpha_{2}\right\}$, positive scalars $\gamma>0$ and $\varepsilon>0$, weighting matrices $\mathscr{Q}, \mathscr{R}$ and matrices $M_{c}(k)=A_{c}(k) P_{1}(k)$, $Y_{F}(k)=F_{x}(k) P_{1}(k)$ such that (8)-(11) are satisfied, the controller parameters $\left\{A_{c}(k), L_{c}(k), F_{x}(k), F_{y}(k)\right\}$ guarantee that $\tilde{x}(i+1 \mid k), i \geq 0$, are RPI in $\mathcal{E}\left(P^{-1}(k)\right)$ thereafter.

$$
\begin{aligned}
& {\left[\begin{array}{cc}
\rho(k) Q_{e}^{-1}(k) & \star \\
I & P_{2}(k)
\end{array}\right] \geq 0,} \\
& {\left[\begin{array}{cc}
1-\rho(k) & \star \\
x_{c}(k) & P_{1}(k)
\end{array}\right] \geq 0,} \\
& \Pi_{l j}^{C}(k, \varepsilon) \geq 0, l=j, l, j \in \mathbb{Z}_{[1, L]}, \\
& \Pi_{l j}^{C}(k, \varepsilon)+\Pi_{j l}^{C}(k, \varepsilon) \geq 0, j>l, \quad l, j \in \mathbb{Z}_{[1, L]}, \\
& 1-\alpha_{1}-\alpha_{2} \geq 0,1 \geq \alpha_{1} \geq 0,1 \geq \alpha_{2} \geq 0,
\end{aligned}
$$

where $\Pi_{l j}^{C}(k, \varepsilon), l, j \in \mathbb{Z}_{[1, L]}$, are given in (10.a).

Proof: For the constraint on $\tilde{x}(k) \in \mathcal{E}\left(P^{-1}(k)\right)$, according to the definitions of the augmented state and $P(k)$,

$$
x_{c}^{\mathrm{T}}(k) P_{1}^{-1}(k) x_{c}(k)+e^{\mathrm{T}}(k) P_{2}^{-1}(k) e(k) \leq 1 .
$$

Let $e^{\mathrm{T}}(k) P_{2}^{-1}(k) e(k) \leq \rho(k) e^{\mathrm{T}}(k) Q_{e}^{-1}(k) e(k) \leq \rho(k)$. Then, (12) amouts to $x_{c}^{\mathrm{T}}(k) P_{1}^{-1}(k) x_{c}(k) \leq 1-\rho(k)$. The above two constraints are respectively guaranteed by (8) and (9). For all possible $\tilde{x}(i \mid k) \in \mathcal{E}\left(P^{-1}(k)\right)$ and $w(k+i) \in$ $\mathcal{E}\left(P_{w}\right), i \geq 0$, by applying the S-procedure, $\tilde{x}(i+1 \mid k) \in$ $\mathcal{E}\left(P^{-1}(k)\right), i \geq 0$, are satisfied if there exist non-negative scalars $\left\{\alpha_{1}, \alpha_{2}\right\}$ and a positive scalar $\gamma>0$ such that

$$
\begin{aligned}
& 1-\|\tilde{x}(i+1 \mid k)\|_{P^{-1}(k)}^{2}-\alpha_{1}\left(1-\|\tilde{x}(i \mid k)\|_{P^{-1}(k)}^{2}\right)-\alpha_{2}(1- \\
& \left.\|w(k+i)\|_{P_{w}}^{2}\right) \geq \frac{1}{\gamma}\left[\|y(i \mid k)\|_{\mathscr{Q}}^{2}+\|u(i \mid k)\|_{\mathscr{R}}^{2}\right]
\end{aligned}
$$

If (11) and (14) are satisfied, then (13) holds.

$$
\begin{aligned}
& \alpha_{1}\|\tilde{x}(i \mid k)\|_{P^{-1}(k)}^{2}+\alpha_{2}\|w(k+i)\|_{P_{w}}^{2}-\|\tilde{x}(i+1 \mid k)\|_{P^{-1}(k)}^{2} \\
& \geq \frac{1}{\gamma}\left[\|y(i \mid k)\|_{\mathscr{Q}}^{2}+\|u(i \mid k)\|_{\mathscr{R}}^{2}\right] .
\end{aligned}
$$

For all $\tilde{x}(i \mid k) \in \mathcal{E}\left(P^{-1}(k)\right)$ and $w(k+i) \in \mathcal{E}\left(P_{w}\right), i \geq 0$, the sufficient and necessary condition for (14) is

$$
\begin{aligned}
& \Phi(k)-\left[\begin{array}{c}
T^{\mathrm{T}}(k, i) \\
H^{\mathrm{T}}(k, i)
\end{array}\right] P^{-1}(k)[T(k, i), H(k, i)] \geq \\
& \frac{1}{\gamma}\left[\overline{C E}^{\mathrm{T}}(k, i) \mathscr{Q} \overline{C E}(k, i)+{\overline{F_{X Y}}}^{\mathrm{T}}(k, i) \mathscr{R} \overline{F_{X Y}}\right],
\end{aligned}
$$




$$
\Pi_{l j}^{C}(k, \varepsilon)=\left[\begin{array}{ccccccccccc}
\alpha_{1} P_{1}(k) & \star & \star & \star & \star & \star & \star & \star & \star & P_{1}(k) & \star \\
0 & \alpha_{1} P_{2}(k) & \star & \star & \star & \star & \star & \star & \star & \star & P_{2}(k) \\
0 & 0 & \alpha_{2} P_{w} & \star & \star & \star & \star & \star & \star & \star & \star \\
M_{c}(k) & 0 & L_{c}(k) E_{j} & P_{1}(k) & \star & \star & \star & \Lambda_{48 j} & \Lambda_{49 j} & \star & \star \\
\Lambda_{51 l} & A_{l} P_{2}(k) & \Lambda_{53 l j} & 0 & P_{2}(k) & \star & \star & \Lambda_{58 l j} & \Lambda_{59 l j} & \star & \star \\
\Lambda_{61 j} & \Lambda_{62 j} & \mathscr{Q}^{1 / 2} E_{j} & 0 & 0 & \gamma I & \star & \star & \star & \star & \star \\
\mathscr{R}^{1 / 2} Y_{F}(k) & 0 & \mathscr{R}^{1 / 2} F_{y}(k) E_{j} & 0 & 0 & 0 & \gamma I & \Lambda_{78 j} & \Lambda_{79 j} & \star & \star \\
0 & 0 & 0 & \star & \star & 0 & \star & \varepsilon^{-1} I & 0 & \star & \star \\
0 & 0 & 0 & \star & \star & 0 & \star & 0 & \varepsilon^{-1} I & \star & \star \\
\star & 0 & 0 & 0 & 0 & 0 & 0 & 0 & 0 & \varepsilon I & \star \\
0 & \star & 0 & 0 & 0 & 0 & 0 & 0 & 0 & 0 & \varepsilon I
\end{array}\right],
$$

$\Lambda_{48 j}=\Lambda_{49 j}=L_{c}(k) C_{j}, \Lambda_{51 l}=A_{l} P_{1}(k)+B_{l} Y_{F}(k)-M_{c}(k), \quad \Lambda_{53 l j}=B_{l} F_{y}(k) E_{j}+D_{l}-L_{c}(k) E_{j}$,

$\Lambda_{58 l j}=\Lambda_{59 l j}=B_{l} F_{y}(k) C_{j}-L_{c}(k) E_{j}, \Lambda_{61 j}=\mathscr{Q}^{1 / 2} C_{j} P_{1}(k), \Lambda_{62 j}=\mathscr{Q}^{1 / 2} C_{j} P_{2}(k), \Lambda_{78 j}=\Lambda_{79 j}=\mathscr{R}^{1 / 2} F_{y}(k) C_{j}$.

where $\Phi(k)=\operatorname{diag}\left\{\alpha_{1} P^{-1}(k), \alpha_{2} P_{w}\right\}, \quad \overline{F_{X Y}}(k, i)=$ $\left[F_{x}(k)+F_{y}(k) C(k, i), F_{y}(k) C(k, i), F_{y}(k) E(k, i)\right], \overline{C E}(k, i)=$ $[C(k, i), C(k, i), E(k, i)]$. By applying the Schur complement and considering the convexity of description of system parameters, (15) is equivalent to

$$
\begin{aligned}
& \widetilde{\Pi}_{l j}^{C}(k)=\left[\begin{array}{cccc}
\alpha_{1} M(k) & \star & \star & \star \\
0 & \alpha_{2} P_{w} & 0 & \star \\
T_{l j} & H_{l j} & P(k) & \star \\
\widetilde{\Delta}_{41 j} & \Delta_{42 j} & 0 & \gamma I
\end{array}\right] \geq 0, \\
& \widetilde{\Delta}_{41 j}=\left[\begin{array}{cc}
\mathscr{Q}^{1 / 2} C_{j} & \mathscr{Q}^{1 / 2} C_{j} \\
\mathscr{R}^{1 / 2}\left[F_{x}(k)+F_{y}(k) C_{j}\right] & \mathscr{R}^{1 / 2} F_{y}(k) E_{j}
\end{array}\right] \text {. } \\
& \Delta_{42 j}=\left[\begin{array}{c}
\mathscr{Q}^{1 / 2} E_{j} \\
\mathscr{R}^{1 / 2} F_{y}(k) E_{j}
\end{array}\right]
\end{aligned}
$$

Pre- and post-multiply the left and right sides of (16) by $\operatorname{diag}\{P(k), I\}$, respectively, and let $M_{c}(k)=A_{c}(k) P_{1}(k)$, $Y_{F}(k)=F_{x}(k) P_{1}(k)$, then $(16)$ is reformulated as

$$
\begin{aligned}
& {\left[\begin{array}{cccc}
\alpha_{1} P(k) & \star & \star & \star \\
0 & \alpha_{2} P_{w} & 0 & \star \\
\widehat{\Delta}_{31 l j} & H_{l j} & P(k) & \star \\
\widehat{\Delta}_{41 j} & \Delta_{42 j} & 0 & \gamma I
\end{array}\right] \geq 0} \\
& \widehat{\Delta}_{31 l j}=\left[\begin{array}{cc}
M_{c}(k)+L_{c}(k) C_{j} P_{1}(k) & L_{c}(k) C_{j} P_{2}(k) \\
\widehat{\Delta}_{31 l j}^{21} & \widehat{\Delta}_{31 l j}^{22}
\end{array}\right] \text {, } \\
& \widehat{\Delta}_{31 l j}^{21}=A_{l} P_{1}(k)+B_{l} F_{y}(k) C_{j} P_{1}(k)-L_{c}(k) C_{j} P_{1}(k) \\
& +B_{l} Y_{F}(k)-M_{c}(k), \quad \widehat{\Delta}_{41 j}^{22}=\mathscr{Q}^{1 / 2} C_{j} P_{2}(k), \\
& \widehat{\Delta}_{31 l j}^{22}=A_{l} P_{2}(k)+B_{l} F_{y}(k) C_{j} P_{2}(k)-L_{c}(k) C_{j} P_{2}(k), \\
& \widehat{\Delta}_{41 j}=\left[\begin{array}{cc}
\mathscr{Q}^{1 / 2} C_{j} P_{1}(k) & \mathscr{Q}^{1 / 2} C_{j} P_{2}(k) \\
\mathscr{R}^{1 / 2}\left[Y_{F}(k)+F_{y}(k) C_{j} P_{1}(k)\right] & \widehat{\Delta}_{41 j}^{22}
\end{array}\right] .
\end{aligned}
$$

Finally, (17) is equivalent to

$$
\Xi_{l j}(k)-X_{l j}^{\mathrm{T}}(k) Y(k)-Y^{\mathrm{T}}(k) X_{l j}(k) \geq 0,
$$

$$
\begin{aligned}
& \Xi_{l j}(k)=\left[\begin{array}{cccc}
\alpha_{1} P(k) & \star & \star & \star \\
0 & \alpha_{2} P_{w} & 0 & \star \\
\Delta_{31 l} & \Delta_{32 l j} & P(k) & \star \\
\Delta_{41 j} & \Delta_{42 j} & 0 & \gamma I
\end{array}\right], \\
& \Delta_{31 l}=\left[\begin{array}{cc}
M_{c}(k) & 0 \\
\Lambda_{51 l} & A_{l} P_{2}(k)
\end{array}\right], \Delta_{32 l j}=\left[\begin{array}{c}
L_{c}(k) E_{j} \\
\Lambda_{53 l j}
\end{array}\right] \\
& \Delta_{41 j}=\left[\begin{array}{cc}
\mathscr{Q}^{1 / 2} C_{j} P_{1}(k) & \mathscr{Q}^{1 / 2} C_{j} P_{2}(k) \\
\mathscr{R}^{1 / 2} Y_{F}(k) & 0(k)
\end{array}\right], \\
& X_{l j}(k)=\left[\begin{array}{ccccccc}
0 & 0 & 0 & \Lambda_{48 j}^{\mathrm{T}} & \Lambda_{58 l j}^{\mathrm{T}} & 0 & \Lambda_{78 j}^{\mathrm{T}} \\
0 & 0 & 0 & \Lambda_{49 j}^{\mathrm{T}} & \Lambda_{59 l j}^{\mathrm{T}} & 0 & \Lambda_{79 j}^{\mathrm{T}}
\end{array}\right], \\
& Y(k)=\left[\begin{array}{ccccccc}
-P_{1}(k) & 0 & 0 & 0 & 0 & 0 & 0 \\
0 & -P_{2}(k) & 0 & 0 & 0 & 0 & 0
\end{array}\right] .
\end{aligned}
$$

By applying the Young's inequality in Lemma 1 and the Schur complement, and considering the quadratic form of the matrix $Y(k),(18)$ is satisfied if $\sum_{l=1}^{L} \sum_{j=1}^{L} \lambda_{l}(k+$ i) $\lambda_{j}(k+i) \Pi_{l j}^{C}(k, \varepsilon) \geq 0$, where $\Pi_{l j}^{C}(k, \varepsilon)$ are given in Theorem 1. By employing "Proposition 2" in [26] (where the complexity parameter $n=2$ ) to deal with the nonnegativity of double convex summations, $\sum_{l=1}^{L} \sum_{j=1}^{L} \lambda_{l}(k+$ i) $\lambda_{j}(k+i) \Pi_{l j}^{C}(k, \varepsilon) \geq 0$ is guaranteed by (10).

\subsection{Constraints on the input and state}

Based on the robust stability of the augmented closedloop system (4), the following Lemma 2 deals with the input and state constraints in (2).

Lemma 2: The input and state constraints in (2) are satisfied if there exist matrices $P_{1}(k), P_{2}(k), M_{c}(k)=$ $A_{c}(k) P_{1}(k), Y_{F}(k)=F_{x}(k) P_{1}(k)$, and non-negative scalars $\left\{\theta_{1}, \theta_{2}, \theta_{3}, \theta_{4}\right\}$, such that (8)-(11) and the following conditions are satisfied:

$$
\Pi_{j s}^{U} \geq 0, s \in \mathbb{Z}_{\left[1, n_{u}\right]}, j \in \mathbb{Z}_{[1, L]},
$$




$$
\begin{aligned}
& \Pi_{j s}^{U}=\left[\begin{array}{ccccc}
\theta_{1} P(k) & \star & \star & \star & P(k) \\
0 & \theta_{2} P_{w} & \star & \star & \star \\
\Pi_{31 s}^{U} & \xi_{s} F_{y}(k) E_{j} & \bar{u}_{s}^{2} & \Pi_{34 j s}^{U} & \star \\
0 & 0 & \star & \varepsilon^{-1} I & \star \\
\star & 0 & 0 & 0 & \varepsilon I
\end{array}\right], \\
& \Pi_{31 s}^{U}=\left[\begin{array}{cccc}
\xi_{s} Y_{F}(k) & 0
\end{array}\right], \Pi_{34 j s}^{U}=\xi_{s}\left[F_{y}(k) C_{j}, F_{y}(k) C_{j}\right], \\
& 1-\theta_{1}-\theta_{2} \geq 0,1 \geq \theta_{1} \geq 0,1 \geq \theta_{2} \geq 0, \\
& \Pi_{l j f}^{S}(k, \varepsilon) \geq 0, l=j, l, j \in \mathbb{Z}_{[1, L]}, \\
& \Pi_{l j f}^{S}(k, \varepsilon)+\Pi_{j l f}^{S}(k, \varepsilon) \geq 0, j>l, l, j \in \mathbb{Z}_{[1, L]},
\end{aligned}
$$

where $\xi_{s}\left(\zeta_{f}\right), s \in \mathbb{Z}_{\left[1, n_{u}\right]}\left(f \in \mathbb{Z}_{\left[1, n_{x}\right]}\right)$, are the $s$-th $(f$-th) row of the $n_{u}$-ordered ( $n_{x}$-ordered) identity matrix.

Proof: The proof is given in Appendix A.

\section{DYNAMIC OFRMPC WITH CONVEX OPTIMIZATION}

4.1. Main optimization problem and bounds of the estimation error set

The following main optimization problem is solved to minimize the scalar $\gamma$ and optimize the dynamic output feedback controller parameters.

$$
\begin{aligned}
& \min _{\alpha_{1}, \alpha_{2}, \theta_{1}, \theta_{2}, \theta_{3}, \theta_{4}, \rho, \gamma, M_{c}, L_{c}, Y_{F}, F_{y}, P_{1}, P_{2}} \gamma, \\
& \text { s.t.(8)-(11), (19)-(22). }
\end{aligned}
$$

Notice that there are some bilinear terms in (17), (A.4), and (A.11), which lead to the difficulties in solving nonconvex BMI optimization problem $[19,20]$. By resorting to the Young's inequality in Lemma 1, the non-convex BMI constraints in (17), (A.4), and (A.11) are respectively reformulated as (10), (19) and (21). When the scalars $\alpha_{1}$, $\theta_{1}, \theta_{3}$ and $\varepsilon$ are pre-specified, the constraints in problem (23)-(24) are LMIs and solved by semi-definite programming, which is a convex optimization and solved via an LMI toolbox.

Suppose that at time $k \geq 0$, the optimal solution to problem (23)-(24) is $\left\{\alpha_{1}, \alpha_{2}, \theta_{1}, \theta_{2}, \theta_{3}, \theta_{4}, \rho, \gamma, M_{c}, L_{c}, Y_{F}, F_{y}, P_{1}\right.$, $\left.P_{2}\right\}^{*}(k)$. Based on Theorem 1, the augmented state at time $k+1$ satisfies $\tilde{x}(k+1) \in \mathcal{E}\left(\left[P^{*}(k)\right]^{-1}\right)$, i.e.,

$$
\left\|x_{c}(k+1)\right\|_{\left[P_{1}^{*}(k)\right]^{-1}}^{2}+\|e(k+1)\|_{\left[P_{2}^{*}(k)\right]^{-1}}^{2} \leq 1 .
$$

In problem (23)-(24), only (8) and (9) are related with the state information. At time $k+1$, choose $\left\{P_{1}(k+1), P_{2}(k+\right.$ $1)\}=\left\{P_{1}^{*}(k), P_{2}^{*}(k)\right\}$ and let

$$
\begin{aligned}
\rho(k+1) & =1-x_{c}^{\mathrm{T}}(k+1)\left[P_{1}(k+1)\right]^{-1} x_{c}(k+1), \\
Q_{e}(k+1) & =\rho(k+1) P_{2}(k+1),
\end{aligned}
$$

the constraints (8) and (9) at time $k+1$ are satisfied. By further choosing $\left\{\alpha_{1}, \alpha_{2}, \theta_{1}, \theta_{2}, \theta_{3}, \theta_{4}, \rho, \gamma, M_{c}, L_{c}, Y_{F}, F_{y}\right\}(k+$ $1)=\left\{\alpha_{1}, \alpha_{2}, \theta_{1}, \theta_{2}, \theta_{3}, \theta_{4}, \rho, \gamma, M_{c}, L_{c}, Y_{F}, F_{y}\right\}^{*}(k)$, problem (23)-(24) is feasible at time $k+1$. Therefore, according to the invariance condition on the augmented closed-loop system, $e(k+1) \in \mathcal{E}\left(Q_{e}^{-1}(k+1)\right)$. Lemma 3 refreshes bounds of the estimation error set at time $k+1$, which bases on the estimation error system and compares with the set $\mathcal{E}\left(Q_{e}^{-1}(k+1)\right)$.

Lemma 3: For systems (1) and (3), suppose that at time $k \geq 0, e(k) \in \mathcal{E}\left(Q_{e}^{-1}(k)\right)$ and $w(k) \in \mathcal{E}\left(P_{w}\right)$. If there exist a symmetric positive matrix $\widehat{Q}_{e}(k+1)$ and non-negative scalars $\left\{\phi_{1}, \phi_{2}\right\}$ such that problem (27)-(29) is feasible, $e(k+1) \in \mathcal{E}\left(\widehat{Q}_{e}^{-1}(k+1)\right)$, else $e(k+1) \in$ $\mathcal{E}\left(Q_{e}^{-1}(k+1)\right)$.

$$
\begin{aligned}
& \min _{\widehat{Q}_{e}(k+1) \geq 0, \phi_{1} \geq 0, \phi_{2} \geq 0} \operatorname{tr}\left(\widehat{Q}_{e}(k+1)\right), \\
& \text { s.t. }\left[\begin{array}{cccc}
\Gamma_{11} & \star & \star & \star \\
0 & \phi_{1} Q_{e}^{-1} & \star & \star \\
0 & 0 & \phi_{2} P_{w} & * \\
\Gamma_{31 l} & A_{l} & D_{l} & \widehat{Q}_{e}(k+1)
\end{array}\right] \geq 0, \\
& Q_{e}(k+1) \geq \widehat{Q}_{e}(k+1) . \\
& \Gamma_{11}=1-\phi_{1}-\phi_{2}, \\
& \Gamma_{31 l}=A_{l} x_{c}(k)-x_{c}(k+1)+B_{l} u(k), l \in \mathbb{Z}_{[1, L]} .
\end{aligned}
$$

Proof: Define $\tilde{\theta}=\left[1, e^{\mathrm{T}}(k), w^{\mathrm{T}}(k)\right]^{\mathrm{T}} \in \mathfrak{R}^{1+n_{x}+n_{w}}$. According to (1) and (3),

$$
\begin{aligned}
& e(k+1)=x(k+1)-x_{c}(k+1) \in \operatorname{Co}\left\{\Pi_{l} \tilde{\theta}(k)\right\}, \\
& \Pi_{l}=\left[A_{l} x_{c}(k)-x_{c}(k+1)+B_{l} u(k), A_{l}, D_{l}\right] .
\end{aligned}
$$

At time $k+1, e(k+1) \in \mathcal{E}\left(\widehat{Q}_{e}^{-1}(k+1)\right)$ is represented by

$$
\tilde{\theta}^{\mathrm{T}} \operatorname{diag}\{1,0,0\} \tilde{\boldsymbol{\theta}}-\tilde{\boldsymbol{\theta}}^{\mathrm{T}} \Pi_{l}^{\mathrm{T}} \widehat{Q}_{e}^{-1}(k+1) \Pi_{l} \tilde{\boldsymbol{\theta}} \geq 0 .
$$

Furthermore, $e^{\mathrm{T}}(k) Q_{e}^{-1}(k) e(k) \leq 1$ and $w^{\mathrm{T}}(k) P_{w} w(k) \leq 1$ can be represented by (32) and (33), respectively.

$$
\begin{aligned}
& \tilde{\theta}^{\mathrm{T}} \operatorname{diag}\left\{1,-Q_{e}^{-1}(k), 0\right\} \tilde{\boldsymbol{\theta}} \geq 0, \\
& \tilde{\theta}^{\mathrm{T}} \operatorname{diag}\left\{1,0,-P_{w}\right\} \tilde{\boldsymbol{\theta}} \geq 0 .
\end{aligned}
$$

By applying the S-procedure, a sufficient condition for "(32) and (33) $\Rightarrow(31)$ " to hold is that there exist nonnegative scalars $\phi_{1}$ and $\phi_{2}$ such that

$$
\begin{aligned}
& \tilde{\theta}^{\mathrm{T}} \operatorname{diag}\{1,0,0\} \tilde{\theta}-\tilde{\theta}^{\mathrm{T}} \Pi_{l}^{\mathrm{T}} \widehat{Q}_{e}^{-1}(k+1) \Pi_{l} \tilde{\theta}-\phi_{1} \tilde{\theta}^{\mathrm{T}} \times \\
& \operatorname{diag}\left\{1,-Q_{e}^{-1}, 0\right\} \tilde{\theta}-\phi_{2} \tilde{\theta}^{\mathrm{T}}(k) \operatorname{diag}\left\{1,0,-P_{w}\right\} \tilde{\theta} \geq 0 . \\
& l \in \mathbb{Z}_{[1, L]} .
\end{aligned}
$$


The necessary and sufficient condition for (34) is

$$
\begin{aligned}
& \operatorname{diag}\{1,0,0\}-\Pi_{l}^{\mathrm{T}} \widehat{Q}_{e}^{-1}(k+1) \Pi_{l}-\phi_{1} \operatorname{diag}\{1, \\
& \left.-Q_{e}^{-1}, 0\right\}-\phi_{2} \operatorname{diag}\left\{1,0,-P_{w}\right\} \geq 0, l \in \mathbb{Z}_{[1, L]} .
\end{aligned}
$$

By applying the Schur complement, (35) is equivalent to (28). If problem (27)-(29) is feasible, a smaller estimation error set $\mathcal{E}\left(\widehat{Q}_{e}^{-1}(k+1)\right)$ satisfying $\mathcal{E}\left(\widehat{Q}_{e}^{-1}(k+1)\right) \subseteq$ $\mathcal{E}\left(Q_{e}^{-1}(k+1)\right)$ is optimized. If problem (27)-(29) is infeasible, at time $k+1, e(k+1) \in \mathcal{E}\left(Q_{e}^{-1}(k+1)\right)$.

\subsection{Overall algorithm for dynamic OFRMPC}

Algorithm 1 synthesizes the convex main optimization problem to optimize the dynamic controller parameters and the refreshment on bounds of the estimation error set.

Algorithm 1: Suppose that $e(0) \in \mathcal{E}\left(Q_{e}^{-1}(0)\right)$, where $Q_{e}(0)$ is known at time $k=0$. Choose $x_{c}(0)$ satisfying $\mathcal{E}\left(x_{c}(0), Q_{e}^{-1}(0)\right) \subseteq \mathbb{S}$. Pre-specify the scalars $\left\{\alpha_{1}, \theta_{1}, \theta_{3}\right\} \in(0,1)$.

At time $k=0$, the gridding method (Remark 5 in [23]) searches the scalar $\varepsilon$ to ensure feasibility of problem (23)-(24). Let the scalars $\Delta_{\varepsilon}=1 / N, N \in \mathbb{Z}_{+}$, and $\kappa_{r}=r \Delta_{\varepsilon}, r \in \mathbb{Z}_{[1, N]}$. Rescale $\varepsilon$ by defining $\kappa=\varepsilon /(1+\varepsilon)$. Then, $\varepsilon_{r}=\kappa_{r} /\left(1-\kappa_{r}\right), r \in \mathbb{Z}_{[1, N]}$. Set $r=1$ and perform steps (1)-(3).

(1) Let $\varepsilon=\varepsilon_{r}$, and solve problem (23)-(24).

(2) If problem (23)-(24) is infeasible and $r<N$, increase $r$ by 1 and return to step (1).

(3) If problem (23)-(24) is feasible, the searched $\varepsilon$ is $\varepsilon^{o}$.

At time $k \geq 0$, fix $\varepsilon=\varepsilon^{\mathrm{o}}$ and perform steps (4)-(7).

(4) Solve problem (23)-(24) to obtain the solution $\left\{\alpha_{1}, \alpha_{2}, \theta_{1}, \theta_{2}, \theta_{3}, \theta_{4}, \rho, \gamma, M_{c}(k), L_{c}(k), Y_{F}(k), F_{y}(k)\right.$, $\left.P_{1}(k), P_{2}(k)\right\}^{*}$.

(5) Calculate $A_{c}^{*}(k)=M_{c}^{*}(k)\left[P_{1}^{*}(k)\right]^{-1}$ and $F_{x}^{*}(k)=$ $Y_{F}^{*}(k)\left[P_{1}^{*}(k)\right]^{-1}$. Implement the control input $u(k)=$ $F_{x}^{*}(k) x_{c}(k)+F_{y}^{*}(k) y(k)$ to system (1), and let $x_{c}(k+$ 1) $=A_{c}^{*}(k) x_{c}(k)+L_{c}^{*}(k) y(k)$.

(6) Calculate the scalar $\rho(k+1)$ and matrix $Q_{e}(k+1)$ according to (26).

(7) If problem (27)-(29) is feasible, let $e(k+1) \in$ $\mathcal{E}\left(\widehat{Q}_{e}^{-1}(k+1)\right)$, else $e(k+1) \in \mathcal{E}\left(Q_{e}^{-1}(k+1)\right)$.

Remark 2: In Algorithm 1, the scalar $\varepsilon$ is off-line searched to ensure feasibility of problem (23)-(24) at time $k=0$. Obviously, the scalars $\varepsilon_{r}>0$ if and only if $\kappa_{r} \in$ $(0,1)$. For each grid points of $\left(\kappa_{1}, \kappa_{2}, \ldots, \kappa_{N}\right)$, we can get a sequence of scalars $\varepsilon_{r}, r \in \mathbb{Z}_{[1, N]}$. It can be seen that when the integer $N$ is larger, the uniform grid on each $\kappa_{r}$ will be smaller, which is advantageous for improving search accuracy on the scalar $\varepsilon$. However, the off-line computation burden on searching the scalar $\varepsilon$ will be larger. Compared with "Algorithm 1 in $[12,14]$ " and the main optimization problem in [13], where an iterative CCL approach problem is applied to optimize dynamic controller parameters, problem (23)-(24) is solved as a non-iterative method and the computational burden can be reduced. Furthermore, by properly updating bounds of the estimation error sets in steps (6) and (7), recursive feasibility of problem (23)(24) can be ensured (see the proof in Theorem 2).

\section{THE COMPARISON WITH DYNAMIC OFRMPC VIA THE CCL APPROACH}

Similar to "Algorithm 1 in $[12,14]$ " and the main optimization problem in [13], the following optimization problem can be solved to optimize the dynamic output feedback controller parameters.

$$
\begin{array}{ll} 
& \min { }_{\alpha_{1}, \alpha_{2}, \theta_{1}, \theta_{2}, \theta_{3}, \theta_{4}, \gamma, A_{c}, L_{c}, F_{x}, F_{y}, P_{1}, P_{2}, M_{1}, M_{2}} \gamma, \\
\text { s.t. } & (8)(9)(11)(20)(22)(\mathrm{A} .4), P(k)=M^{-1}(k), \\
& \widetilde{\Pi}_{l j}^{C}(k) \geq 0, l=j, l, j \in \mathbb{Z}_{[1, L]}, \\
& \widetilde{\Pi}_{l j}^{C}(k)+\widetilde{\Pi}_{j l}^{C}(k) \geq 0, j>l, l, j \in \mathbb{Z}_{[1, L]}, \\
& \widetilde{\Pi}_{l j t}^{S}(k) \geq 0, l=j, l, j \in \mathbb{Z}_{[1, L]}, t \in \mathbb{Z}_{\left[1, n_{x}\right]} \\
& \widetilde{\Pi}_{l j t}^{S}(k)+\widetilde{\Pi}_{j l t}^{S}(k) \geq 0, j>l, l, j \in \mathbb{Z}_{[1, L]},
\end{array}
$$

where $\widetilde{\Pi}_{l j}^{C}(k)$ and $\widetilde{\Pi}_{l j}^{S}(k)$ are respectively in (16) and (A.10). Problem (36)-(39) is non-convex optimization even if $\alpha_{1}, \theta_{1}$ and $\theta_{3}$ are fixed due to inverse matrices $P(k)$ and $M(k)$ [21]. To solve problem (36)-(39), the CCL approach in [12-14] is employed to achieve $P(k)=M^{-1}(k)$ and minimize $\gamma$ by an iterative approach. Firstly, add condition (40) to problem (36)-(39).

$$
\left[\begin{array}{cc}
P(k) & I \\
I & M(k)
\end{array}\right] \geq 0 .
$$

Then, problem (36)-(39) becomes

$$
\begin{aligned}
& \min _{\alpha_{1}, \alpha_{2}, \theta_{1}, \theta_{2}, \theta_{3}, \theta_{4}, \gamma, A_{c}, L_{c}, F_{x}, F_{y}, P_{1}, P_{2}, M_{1}, M_{2}} \gamma, \\
& \text { s.t. }(8)(9)(11)(20)(22)(\text { A.4 } 4)(38)-(40) .
\end{aligned}
$$

In problem (41)-(42), it is possible to achieve $P(k)=M^{-1}(k)$ by minimizing $\operatorname{tr}(P(k) M(k))$ because $\operatorname{tr}(P(k) M(k)) \geq 2 n_{x}$ always satisfies when (40) is considered. The minimization of $\operatorname{tr}(P(k) M(k))$ should accompany the minimization of $\gamma$. Algorithm 2 summarizes the procedures to solve problem (41)-(42) and refresh bounds of the estimation error set.

Algorithm 2: At time $k=0$, select $x_{c}(0)$ and $e(0) \in$ $\mathcal{E}\left(Q_{e}^{-1}(0)\right)$. Pre-specify a larger integer $N_{0} \in \mathbb{Z}_{+}$and scalars $\left\{\alpha_{1}, \theta_{1}, \theta_{3}, \kappa\right\} \in(0,1)$. At time $k \geq 0$, perform the following steps:

(1) Set $t=0$, flag $=0, \gamma^{0}=\infty$. Solve problem (41)-(42) and denote $\{P, M, \rho\}^{t}=\{P, M, \rho\}^{*}$. 
(2) Increase $t$ by 1 and solve

$$
\begin{array}{r}
\min _{\alpha_{2}, \theta_{2}, \theta_{4}, \gamma, \rho, A_{c}, L_{c}, F_{x}, F_{y}} \gamma, \\
\text { s.t. }(8)(9)(11)(20)(22)(\text { A.4 } 4)(38)-(39), \\
\{P, M, \rho\}=\{M, M, \rho\}^{t-1}, \gamma<\kappa \gamma^{0} .
\end{array}
$$

If problem (43)-(44) is feasible, denote $\left\{\gamma, A_{c}, L_{c}, F_{x}, F_{y}\right\}^{\circ}(k)=\left\{\gamma, A_{c}, L_{c}, F_{x}, F_{y}\right\}^{*},\{P, M\}^{\circ}(k)$ $=\left\{\left(M^{t-1}\right)^{-1}, M^{t-1}\right\},\{P, M, \rho\}^{t}=\{P, M, \rho\}^{t-1}$, set flag $=1$ and go to step (4), else solve problem (45)(46) to obtain $\{M, P, \rho\}^{t}=\{M, P, \rho\}^{*}$ and go to step (3).

$$
\begin{aligned}
& \min _{\alpha_{2}, \theta_{2}, \theta_{4}, \gamma, \rho, A_{c}, L_{c}, F_{x}, F_{y}, M_{1}, M_{2}, P_{1}, P_{2}} \operatorname{tr}(\Theta), \\
& \text { s.t. }(8)(9)(11)(20)(22)(\mathrm{A} .4)(38)-(40), \gamma<\kappa \gamma^{0}, \\
& \Theta=P^{t-1} M+M^{t-1} P .
\end{aligned}
$$

(3) If $t<N_{0}$ and flag $=0$, go to step (2).

(4) If $t<N_{0}$ and flag $=1$, set $\{M, P, \rho\}^{0}=\{M, P, \rho\}^{t}$, $t=0$, and go to step (2).

(5) If $t=N_{0}$ and flag $=1$, the final iterative solution to problem (43)-(44) is $\left\{\gamma, A_{c}, L_{c}, F_{x}, F_{y}, M_{1}, M_{2}, P_{1}, P_{2}\right\}^{\circ}(k)$.

(6) Implement $u(k)=F_{x}^{\mathrm{o}}(k) x_{c}(k)+F_{y}^{\mathrm{o}}(k) y(k)$ and let $x_{c}(k+1)=A_{c}^{\mathrm{o}}(k) x_{c}(k)+L_{c}^{\mathrm{o}}(k) y(k)$.

(7) Let $\left\{P_{1}, P_{2}\right\}(k+1)=\left\{P_{1}, P_{2}\right\}^{\circ}(k)$. Select the scalar $\rho(k+1)$ and matrix $Q_{e}(k+1)$ according to (26).

(8) If problem (27)-(29) is feasible, let $e(k+1) \in$ $\mathcal{E}\left(\widehat{Q}_{e}^{-1}(k+1)\right)$, else $e(k+1) \in \mathcal{E}\left(Q_{e}^{-1}(k+1)\right)$.

Remark 3: In Algorithm 2, $N_{0}$ is the maximal number of iterative steps for the CCL method; $t$ gather the counts for the iterative optimization. At each iteration optimization, three cases are involved. For the case (a) in step (3), problems (43)-(44) and (45)-(46) are iteratively solved to find an initial solution to problem (43)-(44). For the case (b) in step (4), a solution to problem (43)-(44) is obtained and the parameter $t$ is reset to 0 , then problems (43)-(44) and (45)-(46) will be iteratively solved in the following procedures to minimize a smaller $\gamma$. For the case (c) in step (5), the iterative CCL approach to minimize $\gamma$ is terminated, then the final solution to problem (43)-(44) is obtained. It can be seen that the iterative optimization terminates when problem (43)-(44) cannot get the solution within $N_{0}$ steps. Therefore, at each time $k$, problems (43)(44) and (45)-(46) will be iteratively solved at least $N_{0}$ times, which increases the on-line computational burden.

\section{COMPLEXITY ANALYSIS}

The complexity analysis (Table 1) for the optimization problems in Algorithms 1 and 2 solved by an LMI tool is polynomial-time, which (regarding the fastest interiorpoint algorithms) is proportional to $\mathfrak{K}^{3} \mathfrak{L}$, where $\mathfrak{K}$ is the number of LMI scalar variables and $\mathfrak{L}$ is the number of LMI rows [27]. In Algorithm 1, at each time $k$, the convex optimization problem (23)-(24) is only solved one time to simultaneously minimize the scalar $\gamma$ with the corresponding optimal dynamic output feedback controller parameters. In Algorithm 2, considering the existence of mutual inverse matrices $P(k)$ and $M(k)$ in the optimization problem, at each time $k \geq 0$, the iterative CCL approach optimizes the mutual inverse matrices $P(k)$ and $M(k)$ and minimizes the scalar $\gamma$ by an iterative method, where the following steps iterate in finite steps. Firstly, find mutual inverse matrices $P(k)$ and $M(k)$ satisfying $P(k)=M^{-1}(k)$ by an iterative optimization; secondly, minimize the scalar $\gamma$ to optimize the dynamic output controller parameters with the satisfaction of $P(k)=M^{-1}(k)$; thirdly, find mutual inverse matrices $P(k)$ and $M(k)$ again with the additional consideration of the decrease in the scalar $\gamma$. In Table 1, the optimization problems in the compared algorithms have the similar LMI scalar variables and LMI rows. Because the optimization problem in Algorithm 2 takes the iterative optimization, the computational time will increase compared with Algorithm 1.

\section{RECURSIVE FEASIBILITY AND ROBUST STABILITY}

Theorem 2: For the LPV system (1) with bounded disturbances, the dynamic OFRMPC approaches in Algorithms 1 and 2 are performed. If problem (23)-(24) (or (36)-(39)) is feasible at time $k=0$, then recursive feasibility of problem (23)-(24) (or (36)-(39)) is ensured. The optimized dynamic output feedback controller parameters steer the augmented closed-loop system (4) to converge to a neighborhood of the origin such that robust stability of the controlled system (1) is guaranteed. The input and state constraints in (2) are satisfied for all time $k \geq 0$.

Proof: Recursive feasibility of the optimization problem means that once the optimization problem is feasible at time $k=0$, it will be feasible for all time $k \geq 0$. In Algorithm 1, suppose that at time $k \geq 0$, the optimal solution to problem (23)-(24) is $\Gamma^{*}(k)=$ $\left\{\alpha_{1}, \alpha_{2}, \varepsilon, \theta_{1}, \theta_{2}, \theta_{3}, \theta_{4}, \rho, \gamma, M_{c}, L_{c}, Y_{F}, F_{y}, P_{1}, P_{2}\right\}^{*}(k)$. As the analysis in Section 4.1, at time $k+1$, by choosing $\rho(k+1)$ and $Q_{e}(k+1)$ according to (26), and let $\left\{\alpha_{1}, \alpha_{2}, \varepsilon, \theta_{1}, \theta_{2}, \theta_{3}, \theta_{4}, \gamma, M_{c}, L_{c}, Y_{F}, F_{y}\right\}(k+1)=$ $\left\{\alpha_{1}, \alpha_{2}, \varepsilon, \theta_{1}, \theta_{2}, \theta_{3}, \theta_{4}, \gamma, M_{c}, L_{c}, Y_{F}, F_{y}\right\}^{*}(k), \quad$ problem (23)-(24) is feasible at time $k+1$. If problem (27)-(29) is feasible, a smaller estimation error set $\mathcal{E}\left(\widehat{Q}_{e}^{-1}(k+1)\right)$ satisfying $\mathcal{E}\left(\widehat{Q}_{e}^{-1}(k+1)\right) \subseteq \mathcal{E}\left(Q_{e}^{-1}(k+1)\right)$ can be obtained. Replace the matrix $Q_{e}(k+1)$ in (8) by $\widehat{Q}_{e}(k+1)$, problem (23)-(24) will also be feasible at time $k+1$. Therefore, the optimal solutions to problems (23)-(24) and (27)-(29) at time $k$ are a feasible solution to problem (23)-(24) at time $k+1$ such that recursive feasibility of problem (23)-(24) 
Table 1 . The comparison of complexity analysis

\begin{tabular}{|c|c|}
\hline the optimization problems & the scalars $\mathfrak{K}$ and $\mathfrak{L}$ \\
\hline problem (23)-(24) & $\begin{array}{l}\mathfrak{K}=5+n_{x}^{2}+n_{x} n_{y}+n_{u} n_{x}+n_{u} n_{y}+n_{x}\left(n_{x}+1\right), \mathfrak{L}=3 n_{x}+\frac{L(L+1)}{2}\left[8 n_{x}+n_{w}+n_{y}+n_{u}+n_{x}\left(3 n_{x}+\right.\right. \\
\left.\left.n_{w}+1\right)\right]+L n_{u}\left(3 n_{x}+n_{w}+1\right)+4\end{array}$ \\
\hline problem (43)-(44) & $\begin{array}{l}\mathfrak{K}=5+n_{x}^{2}+n_{x} n_{y}+n_{u} n_{x}+n_{u} n_{y}, \mathfrak{L}=3 n_{x}+\frac{L(L+1)}{2}\left[4 n_{x}+n_{w}+n_{y}+n_{u}+n_{x}\left(2 n_{x}+n_{w}+1\right)\right]+ \\
L n_{u}\left(n_{x}+n_{w}+1\right)+5\end{array}$ \\
\hline problem (45)-(46) & $\begin{array}{l}\mathfrak{K}=5+n_{x}^{2}+n_{x} n_{y}+n_{u} n_{x}+n_{u} n_{y}+2 n_{x}\left(n_{x}+1\right), \mathfrak{L}=5 n_{x}+\frac{L(L+1)}{2}\left[4 n_{x}+n_{w}+n_{y}+n_{u}+n_{x}\left(2 n_{x}+\right.\right. \\
\left.\left.n_{w}+1\right)\right]+L n_{u}\left(n_{x}+n_{w}+1\right)+5\end{array}$ \\
\hline
\end{tabular}

is ensured. Here we omit the proof on recursive feasibility of problem (36)-(39) for brevity because the proof is similar. When problem (23)-(24) is solved at time $k+1$, $\gamma^{*}(k+1) \leq \gamma^{*}(k)$ will be obtained. With the evolution of time, $\gamma^{*}(k)$ will converge to a constant value.

Consider the following disturbance-free augmentedclosed loop system (i.e., system (4) without consideration of bounded disturbances), where $\tilde{x}_{u}(k)=\left[x_{c u}^{\mathrm{T}}(k), e_{u}^{\mathrm{T}}(k)\right]^{\mathrm{T}}$.

$$
\begin{aligned}
& \tilde{x}_{u}(i+1 \mid k)=T(k, i) \tilde{x}_{u}(i \mid k), i \geq 0, \tilde{x}_{u}(0 \mid k)=\tilde{x}(k), \\
& y_{u}(i \mid k)=C(k)\left[x_{c u}(i \mid k)+e_{u}(i \mid k)\right] \\
& u_{u}(i \mid k)=F_{x}(k) x_{c u}(i \mid k)+F_{y}(k) y_{u}(i \mid k) .
\end{aligned}
$$

In Theorem 1, condition (10) guarantees the satisfaction of (18). Accordingly, if (18) is satisfied, then (16) holds. By applying the Schur complement, (16) also implies that

$$
\begin{aligned}
& \alpha_{1} P^{-1}(k)-T^{\mathrm{T}}(k, i) P^{-1}(k) T(k, i) \geq \frac{1}{\gamma^{*}(k)} \times \\
& {\left[\overline{C E}^{\mathrm{T}}(k, i) \mathscr{Q} \overline{C E}(k, i)+\bar{F}_{X Y}^{\mathrm{T}}(k, i) \mathscr{R} \bar{F}_{X Y}(k, i)\right],} \\
& \overline{C E}(k, i)=[C(k, i), C(k, i)], \\
& \bar{F}_{X Y}(k, i)=\left[F_{x}(k)+F_{y}(k) C(k, i), F_{y}(k) C(k, i)\right] .
\end{aligned}
$$

Since $\alpha_{1} \in(0,1)$ is pre-specified, (48) ensures that

$$
\begin{aligned}
& P^{-1}(k)-T^{\mathrm{T}}(k, i) P^{-1}(k) T(k, i)>\frac{1}{\gamma^{*}(k)}\left[\overline{C E}^{\mathrm{T}}(k, i) \times\right. \\
& \left.\mathscr{Q} \overline{C E}(k, i)+\bar{F}_{X Y}^{\mathrm{T}}(k, i) \mathscr{R} \bar{F}_{X Y}(k, i)\right], i \geq 0 .
\end{aligned}
$$

The above condition (49) guarantees the stability of the disturbance-free augmented-closed loop system (47), i.e.,

$$
\begin{aligned}
& \left\|\tilde{x}_{\mathrm{u}}(i \mid k)\right\|_{P^{-1}(k)}^{2}-\left\|\tilde{x}_{\mathrm{u}}(i+1 \mid k)\right\|_{P^{-1}(k)}^{2}>\frac{1}{\gamma^{*}(k)} \times \\
& {\left[\left\|y_{\mathrm{u}}(i \mid k)\right\|_{\mathscr{Q}}^{2}+\left\|u_{\mathrm{u}}(i \mid k)\right\|_{\mathscr{R}}^{2}\right], i \geq 0 .}
\end{aligned}
$$

Therefore, (49) guarantees that the disturbance-free augmented state converges within the set $\mathcal{E}\left(P^{-1}(k)\right)$. By summing (50) from $i=0$ to $i=\infty$, then considering $\tilde{x}_{\mathrm{u}}(0 \mid k)=$ $\tilde{x}(k)$ and $\tilde{x}_{\mathrm{u}}(0 \mid k) \in \mathcal{E}\left(P^{-1}(k)\right)$, it can be obtained that

$$
J_{\infty}(k)=\sum_{i=0}^{\infty}\left[\left\|y_{\mathrm{u}}(i \mid k)\right\|_{\mathscr{Q}}^{2}+\left\|u_{\mathrm{u}}(i \mid k)\right\|_{\mathscr{R}}^{2}\right]<\gamma^{*}(k) .
$$

Here, $\gamma^{*}(k)$ is an upper bound of the performance cost for system (47). The condition $J_{\infty}(k)<\gamma^{*}(k)$ results in $\lim _{i \rightarrow \infty}\left\{y_{\mathrm{u}}(i \mid k), u_{\mathrm{u}}(i \mid k)\right\}=\{0,0\}$. With the evolution of time, the disturbance-free system outputs and inputs will converge to the origin. Since bounded disturbances are considered, the augmented closed-loop system (4) will be stabilized within a region in a neighborhood of the origin such that robust stability of the controlled system (1) is ensured. Satisfaction of input and state constraints are due to (19)-(22).

\section{SIMULATION EXAMPLE}

In this section, we provide two simulation examples. The first example is the mass-spring-damping mechanical system, and the second example is a numerical example. For the two simulations examples, we compare Algorithms 1 and 2. In Algorithm 2, select $N_{0}=50$ and $\kappa=0.98$. In Algorithms 1 and 2, choose parameters $\alpha_{1}=\theta_{1}=\theta_{3}=0.98, P_{w}=25$, weight matrices $\mathscr{Q}=25$, $\mathscr{R}=1$. Matlab 9.3 (Intel i5-7200U 2.5GHz, 8G Memory) is utilized for the simulations.

\subsection{Example 1}

According to Newton's law, the mass-spring-damping mechanical system in Figure 1 is described by

$$
m \ddot{x}+F_{f}+F_{s}=u(k),
$$

where $m$ stands for the mass, $F_{f}$ is the friction force, $F_{s}$ is the restoring force of the spring. The friction force $F_{f}=c \dot{x}$ with $c>0$; the hardening spring force $F_{s}=k_{0}\left(1+a^{2} x^{2}\right) x$ with constant parameters $a$ and $k_{0}$. Being different from [28], where the parameter $k_{0}$ is uncertain in an interval set, we assume that the scalar $k_{0}=8 \mathrm{~N} / \mathrm{m}$ is a constant value. Let $x$ denote the spring's displacement from a reference point. Thus,

$$
m \ddot{x}+c \dot{x}+k_{0} x+k_{0} a^{2} x^{3}=u(k) .
$$

Define $x(k)=\left[x_{1}(k), x_{2}(k)\right]^{\mathrm{T}}=[x(k), \dot{x}(k)]^{\mathrm{T}}$. Let $x_{1}(k) \in$ $[-2,2], \quad u(k) \in[-2,2], \quad m=1 \mathrm{~kg}, \quad a=0.3 m^{-1}, \quad c=$ $2 N . \mathrm{m} / \mathrm{s}$. System (52) is reformulated as the following continuous-time state space model, where bounded disturbance $w(k) \in \varepsilon_{P_{w}}$ is additional considered.

$$
\dot{x}(k)=A^{c}(k) x(k)+B^{c}(k) u(k)+D^{c}(k) w(k),
$$




$$
y(k)=C^{c}(k) x(k)+E^{c}(k) w(k),
$$

where $A^{c}(k)=\left[\begin{array}{cc}0 & 1 \\ -\frac{k_{0}+k_{0} a^{2} x_{1}^{2}(k)}{m} & -\frac{c}{m}\end{array}\right], B^{c}(k)=\left[\begin{array}{c}0 \\ \frac{1}{m}\end{array}\right]$, $C^{c}(k)=[1,0], \quad D^{c}(k)=[0.01,0.01]^{\mathrm{T}}, \quad E^{c}(k)=0.05$. Here, the matrices with the subscript "c" stand for the continuous-time system parameters. Considering the term “ $-\frac{k_{0}+k_{0} a^{2} x_{1}^{2}(k)}{m}$ " in system matrix $A^{c}(k)$ is nonlinear and the constraints on the state $x_{1}(k)$, by applying the sector nonlinearity method [29], the nonlinear system (53) can be approximated by the convex combination of continuoustime LPV system's sub-models, i.e.,

$$
\begin{aligned}
& \dot{x}(k)=\sum_{l=1}^{L} \lambda_{l}(k)\left[A_{l}^{c} x(k)+B_{l}^{c} u(k)+D_{l}^{c} w(k)\right], \\
& y(k)=\sum_{l=1}^{L} \lambda_{l}(k)\left[C_{l}^{c} x(k)+E_{l}^{c} w(k)\right], l \in \mathbb{Z}_{[1,2]}, \\
& A_{1}^{c}=\left[\begin{array}{cc}
0 & 1 \\
-\frac{k_{0}+4 k_{0} a^{2}}{m} & -\frac{c}{m}
\end{array}\right], A_{2}^{c}=\left[\begin{array}{cc}
0 & 1 \\
-\frac{k_{0}}{m} & -\frac{c}{m}
\end{array}\right], \\
& B_{l}^{c}=\left[\begin{array}{c}
0 \\
\frac{1}{m}
\end{array}\right], C_{l}^{c}=[1,0], D_{l}^{c}=[0.01,0.01]^{\mathrm{T}}, \\
& E_{l}^{c}=0.05, l \in \mathbb{Z}_{[1,2]}, \lambda_{1}(k)=\frac{x_{1}^{2}(k)}{4}, \lambda_{2}(k)=1-\frac{x_{1}^{2}(k)}{4} .
\end{aligned}
$$

Since the state $x_{1}(k)$ is unmeasurable, the non-negative combining coefficients $\lambda_{1}(k)$ and $\lambda_{2}(k)$ of the LPV system are not exactly known. Use the first order Euler approximation to discretize the continuous-time system (54) with sampling period $T_{s}=0.1 \mathrm{~s}$, the corresponding discretetime LPV system parameters in system (1) are obtained.

In the simulation, for Algorithms 1-2, choose $Q_{e}^{-1}(0)=$ $\operatorname{diag}\left\{\frac{0.5}{0.15^{2}}, \frac{0.5}{0.3^{2}}\right\}, e(0)=[0.15,-0.3]^{\mathrm{T}}, x_{c}(0)=[1.5,-3]^{\mathrm{T}}$ and $x(0)=[1.65,-3.3]^{\mathrm{T}}$. For Algorithm 1, at time $k=0$, choose $N=1000$. The searched scalar $\varepsilon^{0}=89.9091$. The responses of the estimated states and true states with the corresponding estimation error sets are shown in Figures 2 and 3 (where the solid (dash) lines with legends are the true (estimated) states, and the ellipsoidal set are the estimation error sets). In Figures 2 and 3, the estimated states converge to the origin, and the true states contained in the ellipsoids with the centers of the estimated states are steered to the neighborhood of origin. The responses of $x_{c}(k)$ and $x(k)$ for Algorithms 1 and 2 in Figure 4 are almost the same. Figure 5 indicates that the input constraints are satisfied. The average computational time for three times simulations that spent on Algorithm 1 and Algorithm 2 are respectively 38.36 seconds and $1569.95 \mathrm{sec}-$ onds. Compared with Algorithm 2, Algorithm 1 significantly reduces the on-line computational burden.

\subsection{Example 2}

Consider the system $A(k)=\left[\begin{array}{cc}0.385 & 0.33 \\ 0.21+\mu(k) & 0.59\end{array}\right]$, where $\mu(k)$ is an uncertain scalar satisfying $|\mu(k)| \leq \bar{\mu}$,

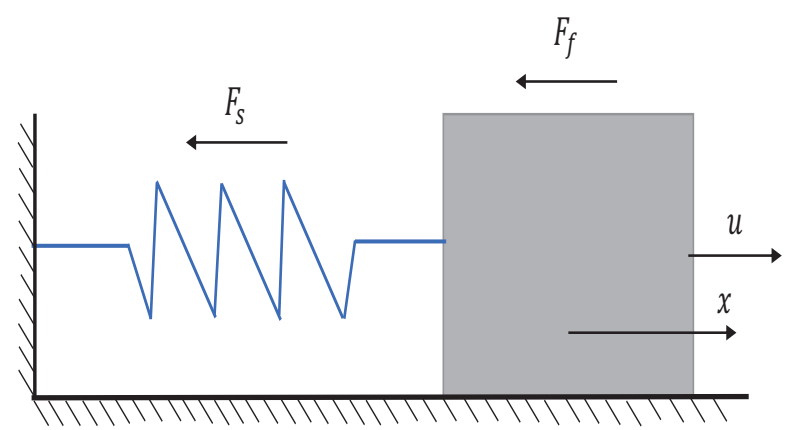

Fig. 1. The mass-spring-damping mechanical system.

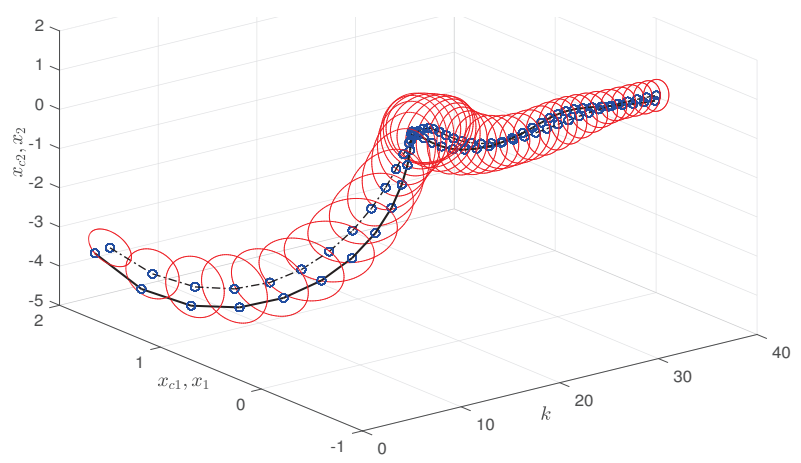

Fig. 2. The responses of $x_{c}(k), x(k)$ and $e(k)$, Algorithm 1, Example 1.

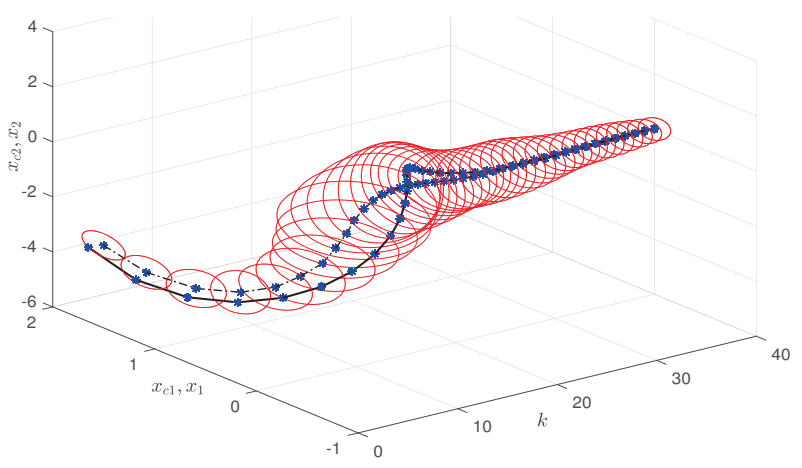

Fig. 3. The responses of $x_{c}(k), x(k)$ and $e(k)$, Algorithm 2, Example 1.

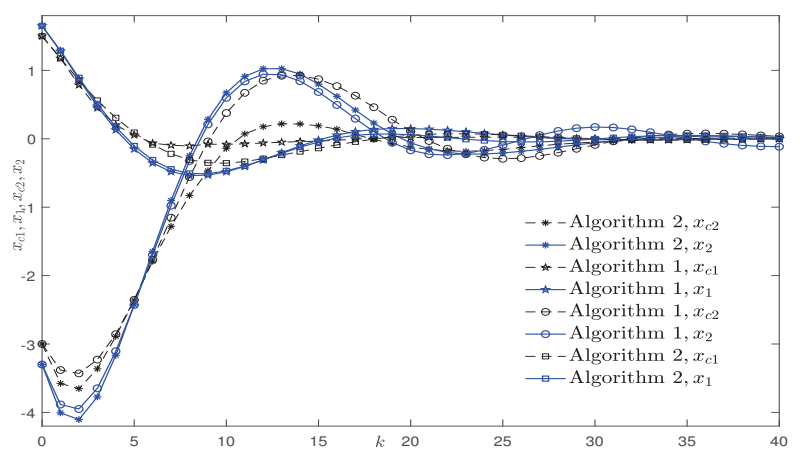

Fig. 4. The comparison on responses of $x_{c}(k)$ and $x(k)$, Example 1. 
Table 2. The simulation time for different bounds of the parameters $|\mu(k)| \leq \bar{\mu}$

\begin{tabular}{|c|l|l|l|l|l|l|}
\hline $\bar{\mu}$ & 0.11 & 0.09 & 0.07 & 0.05 & 0.03 & 0.01 \\
\hline Simulation time for Algorithm 1 (seconds ) & 22.38 & 21.45 & 21.70 & 21.55 & 21.29 & 21.11 \\
\hline Simulation time for Algorithm 2 (seconds ) & 1017.64 & 1024.97 & 1111.40 & 1141.65 & 825.39 & 794.90 \\
\hline
\end{tabular}

$B(k)=\left[\begin{array}{ll}1, & 0\end{array}\right]^{\mathrm{T}}, C(k)=\left[\begin{array}{ll}0, & 1\end{array}\right], \quad D(k)=\left[\begin{array}{ll}0.3, & 0.3\end{array}\right]^{\mathrm{T}}$, and $E(k)=1, \bar{\psi}=24 . \quad \lambda_{1}(k)=\frac{0.5-\mu(k)}{0.5}, \lambda_{2}(k)=\frac{\mu(k)}{0.5}$. $\bar{u}=2$. For Algorithms 1-2, choose $\bar{\mu}=0.11, \mu(k)=$ $\sin (k), Q_{e}^{-1}(0)=\operatorname{diag}\left\{\frac{0.5}{2^{2}}, \frac{0.5}{2^{2}}\right\}, e(0)=[2,2]^{\mathrm{T}}, x_{c}(0)=$ $[13.9500,13.9500]^{\mathrm{T}}$, and $x(0)=[15.9500,15.9500]^{\mathrm{T}}$. In Algorithm 1, choose $N=10000$ and the searched scalar $\varepsilon^{0}=1110.011$. Figures 6 and 7 are the responses of the estimated states and true states with the corresponding estimation error sets (where the solid (dash) lines with legends are the true (estimated) states, and the ellipsoidal set are the estimation error sets). In Figures 8 and 11, the responses of $x_{c}(k)$ and $x(k)$ in the compared algorithms are almost the same. The input constraints shown in Figure 12 are satisfied. Considering that different bounds of LPV system' parameters may have effects on the computational time, Table 2 compares the simulation time for different bounds of parameter $|\mu(k)|$, where the average computational time for three times simulations are considered. It can be seen that the computational time for Algorithm 1 is about 21 seconds. Different bounds of $|\mu(k)|$ can affect the simulation time on Algorithm 2. Nevertheless, similar to Example 1, Algorithm 1 compared with Algorithm 2 reduces the on-line computational burden.

\section{CONCLUSION}

A convexity approach to dynamic OFRMPC is proposed for LPV systems with unknown non-negative combining coefficients and bounded disturbances. The off-line optimization problem searches some parameters to ensure initial feasibility of the on-line main optimization problem such that the dynamic output feedback controller parameters can be simultaneously optimized via the convex optimization problem. The auxiliary optimization problem refreshes bounds of the estimation error set to ensure recursive feasibility of the optimization problem. Compared with the dynamic OFRMPC with the CCL approach, the dynamic OFRMPC with the convex optimization significantly reduces the on-line computational burden.

\section{APPENDIX A}

\section{A.1. Proof of Lemma 2}

The input constraints $-\bar{u} \leq u(i \mid k) \leq \bar{u}$ amount to $\bar{u}_{s}^{2} \geq$ $\left\|\xi_{s} u(i \mid k)\right\|_{I}^{2}, i \geq 0$, i.e., $1-\left\|\xi_{s} u(i \mid k)\right\|_{\left[1 / \bar{u}_{s}^{2}\right]}^{2} \geq 0, s \in \mathbb{Z}_{\left[1, n_{u}\right]}$. Since $\tilde{x}(i \mid k) \in \varepsilon_{P^{-1}(k)}$ and $w(k+i) \in \mathcal{E}\left(P_{w}\right), i \geq 0$, according to the $S$ procedure, the constraints on $1-\left\|\xi_{s} u(i \mid k)\right\|_{\left[1 / \bar{u}_{s}^{2}\right]}^{2}, s \in \mathbb{Z}_{\left[1, n_{u}\right]}$, are satisfied if there exist non-negative scalars $\left\{\theta_{1}, \theta_{2}\right\}$ such that

$$
\begin{aligned}
& 1-\left\|\xi_{s} u(i \mid k)\right\|_{\left[1 / \bar{u}_{s}^{2}\right]}^{2}-\theta_{1}\left(1-\|\tilde{x}(i \mid k)\|_{\left[P^{-1}(k)\right]}^{2}\right)- \\
& \theta_{2}\left(1-\|w(k+i)\|_{P_{w}}^{2}\right) \geq 0, \quad s \in \mathbb{Z}_{\left[1, n_{x}\right]} .
\end{aligned}
$$

A sufficient condition for (A.1) is that conditions (20) and (A.2) simultaneously hold.

$$
\theta_{1}\|\tilde{x}(i \mid k)\|_{P^{-1}(k)}^{2}+\theta_{2}\|w(k+i)\|_{P_{w}}^{2}-\left\|\xi_{s} u(i \mid k)\right\|_{\left[1 / \bar{u}_{s}^{2}\right]}^{2} .
$$

Considering that $u(i \mid k)=\Delta_{\tilde{x}}(k, i) \tilde{x}(i \mid k)+\Delta_{w}(k, i) w(k+i)$, where $\Delta_{\tilde{x}}(k, i)=\left[F_{x}+F_{y} C(k, i), F_{y} C(k, i)\right]$ and $\Delta_{w}(k, i)=F_{y} E(k, i)$, the sufficient and necessary conditions for (A.2) are

$$
\begin{aligned}
& {\left[\begin{array}{cc}
\theta_{1} P^{-1} & 0 \\
0 & \theta_{2} P_{w}
\end{array}\right]-\left[\Delta_{s}^{u}(k, i)\right]^{\mathrm{T}} \frac{1}{\bar{u}_{s}^{2}}\left[\Delta_{s}^{u}(k, i)\right] \geq 0,} \\
& \Delta_{s}^{u}(k, i)=\xi_{s}\left[\Delta_{\tilde{x}}(k, i), \Delta_{w}(k, i)\right], s \in \mathbb{Z}_{\left[1, n_{u}\right]} .
\end{aligned}
$$

By applying the Schur complement and considering the polytopic description of system parameters, (A.3) is equivalent to

$$
\begin{aligned}
& \widetilde{\Pi}_{s j}^{U}=\left[\begin{array}{ccc}
\theta_{1} M & \star & \star \\
0 & \theta_{2} P_{w} & \star \\
\xi_{s} \Delta_{\tilde{x}}^{j} & \xi_{s} \Delta_{w}^{j} & \bar{u}_{s}^{2}
\end{array}\right] \geq 0, j \in \mathbb{Z}_{[1, L]} . \\
& \Delta_{\tilde{x}}^{j}=\left[F_{x}+F_{y} C_{j}, F_{y} C_{j}\right], \Delta_{w}^{j}=F_{y} E_{j}
\end{aligned}
$$

By applying the congruent transformation via $\operatorname{diag}\{P, I\}$, (A.4) holds if

$$
\begin{aligned}
& {\left[\begin{array}{ccc}
\theta_{1} P & \star & \star \\
0 & \theta_{2} P_{w} & \star \\
\xi_{s} \widetilde{\Delta}_{\tilde{x}}^{j} & \xi_{s} F_{y} E_{j} & \frac{1}{2} \bar{u}_{s}^{2}
\end{array}\right] \geq 0, \quad j \in \mathbb{Z}_{[1, L]},} \\
& \widetilde{\Delta}_{\tilde{x}}^{j}=\left[Y_{F}(k)+F_{y} C_{j} P_{1}(k), F_{y} C_{j} P_{2}(k)\right] .
\end{aligned}
$$

The above (A.5) can be reformulated as

$$
\begin{aligned}
& \Theta_{j s}^{U}-\widehat{X}_{j s}^{\mathrm{T}} \widehat{Y}-\widehat{Y}^{\mathrm{T}} \widehat{X}_{j s} \geq 0 \\
& \Theta_{j s}^{U}=\left[\begin{array}{ccc}
\theta_{1} P & \star & \star \\
0 & \theta_{2} P_{w} & \star \\
\xi_{s}\left[Y_{F}, 0\right] & \xi_{s} F_{y} E_{j} & \frac{1}{2} \bar{u}_{s}^{2}
\end{array}\right], \\
& \widehat{X}_{j s}=\left[\begin{array}{llll}
0 & 0 & 0 & {\left[\Pi_{34 j s}^{U}\right]^{\mathrm{T}}}
\end{array}\right], \widehat{Y}=\left[\begin{array}{llll}
-P & 0 & 0 & 0
\end{array}\right] .
\end{aligned}
$$

Similar to Theorem 1, by applying the Young's inequality in (5) and the Schur complement, then further considering the quadratic form of the matrix $\widehat{Y}(k)$, (A.6) is guaranteed by (19).

The state constraints $-\bar{\psi} \leq x(i+1 \mid k) \leq \bar{\psi}$ amount to $\bar{\psi}_{f}^{2} \geq$ $\left\|\xi_{f} x(i+1 \mid k)\right\|_{I}^{2}, i \geq 0$, i.e., $1-\left\|\xi_{f} x(i+1 \mid k)\right\|_{\left[1 / \bar{\psi}_{f}^{2}\right]}^{2} \geq 0, f \in \mathbb{Z}_{\left[1, n_{x}\right]}$. Since $\tilde{x}(i \mid k) \in \varepsilon_{P^{-1}(k)}$ and $w(k+i) \in \mathcal{E}\left(P_{w}\right), i \geq 0$, according to the S-procedure, the constraints on $1-\left\|\xi_{f} x(i+1 \mid k)\right\|_{\left[1 / \bar{\psi}_{f}^{2}\right]}^{2}, f \in$ $\mathbb{Z}_{\left[1, n_{x}\right]}$, are satisfied if there exist non-negative scalars $\left\{\theta_{3}, \theta_{4}\right\}$ such that

$$
\begin{aligned}
& 1-\left\|\xi_{f} x(i+1 \mid k)\right\|_{\left[1 / \bar{\psi}_{t}^{2}\right]}^{2}-\theta_{3}\left(1-\|\tilde{x}(i \mid k)\|_{P^{-1}(k)}^{2}\right)- \\
& \theta_{4}\left(1-\|w(k+i)\|_{P_{w}}^{2}\right) \geq 0, \quad f \in \mathbb{Z}_{\left[1, n_{x}\right]} .
\end{aligned}
$$

A sufficient condition for (A.7) is that conditions (22) and (A.8) simultaneous hold.

$$
\begin{aligned}
& \theta_{3}\|\tilde{x}(i \mid k)\|_{P^{-1}(k)}^{2}+\theta_{4}\|w(k+i)\|_{P_{w}}^{2}-\left\|\xi_{f} x(i+1 \mid k)\right\|_{\left[1 / \bar{\psi}_{f}^{2}\right]}^{2}, \\
& f \in \mathbb{Z}_{\left[1, n_{x}\right]} .
\end{aligned}
$$


The sufficient and necessary conditions for (A.8) are

$$
\begin{aligned}
& {\left[\begin{array}{cc}
\theta_{3} P^{-1} & 0 \\
0 & \theta_{4} P_{w}
\end{array}\right]-\Pi^{\mathrm{T}}(k, i) \frac{1}{\bar{\psi}_{f}^{2}} \Pi(k, i) \geq 0,} \\
& \Pi(k, i)=[[I, I] T(k, i),[I, I] H(k, i)], f \in \mathbb{Z}_{\left[1, n_{x}\right]} .
\end{aligned}
$$

By applying the Schur complement and considering the polytopic of the system parameters, (A.9) is equivalent to

$$
\begin{aligned}
\widetilde{\Pi}_{l j f}^{S} & =\left[\begin{array}{cccc}
\theta_{3} M_{1} & \star & \star & \star \\
0 & \theta_{3} M_{2} & \star & \star \\
0 & 0 & \theta_{4} P_{w} & \star \\
\Xi_{41 l j f} & \Xi_{42 l j f} & \Xi_{43 l j f} & \bar{\psi}_{f}^{2}
\end{array}\right] \geq 0, \\
\Xi_{41 l j f} & =\zeta_{f}\left[A_{l}+B_{l} F_{y} C_{j}+B_{l} F_{x}\right], \Xi_{42 l j f}=\zeta_{f}\left[A_{l}+B_{l} F_{y} C_{j}\right], \\
\Xi_{43 l j f} & =\zeta_{f}\left[B_{l} F_{y} E_{j}+D_{l}\right] .
\end{aligned}
$$

Further by applying the congruent transformation via $\operatorname{diag}\{P, I\}$, (A.10) is guaranteed by

$$
\begin{aligned}
& {\left[\begin{array}{cccc}
\theta_{3} P_{1} & \star & \star & \star \\
0 & \theta_{3} P_{2} & \star & \star \\
0 & 0 & \theta_{4} P_{w} & \star \\
\zeta_{f} \Xi_{41 l j f}^{\prime} & \zeta_{f} \Xi_{42 l j f}^{\prime} & \zeta_{f} \Xi_{43 l j f} & \bar{\psi}_{f}^{2}
\end{array}\right] \geq 0,} \\
& \Xi_{41 l j f}^{\prime}=\zeta_{f}\left[A_{l} P_{1}+B_{l} F_{y} C_{j} P_{1}+B_{l} Y_{F}\right], \\
& \Xi_{42 l j f}^{\prime}=\zeta_{f}\left[A_{l} P_{2}+B_{l} F_{y} C_{j} P_{2}\right] .
\end{aligned}
$$

Condition (A.11) can be reformulated as

$$
\begin{aligned}
& \Theta_{l j f}^{S}- \widetilde{X}_{l j f}^{\mathrm{T}} \widehat{Y}-\widetilde{X}_{l j f}^{\mathrm{T}} \widehat{Y} \geq 0, \\
& \Theta_{l j f}^{S}= {\left[\begin{array}{cccc}
\theta_{3} P_{1} & \star & \star & \star \\
0 & \theta_{3} P_{2} & \star & \star \\
0 & 0 & \theta_{4} P_{w} & \star \\
\bar{\Xi}_{41 l f} & \zeta_{f} A_{l} P_{2} & \Xi_{43 l j f} & \bar{\psi}_{f}^{2}
\end{array}\right], } \\
&\left.\bar{\Xi}_{41 l f}=\zeta_{f}\left[A_{l} P_{1}+B_{l} Y_{F}\right], \widetilde{X}_{l j f}=\left[\begin{array}{llll}
0 & 0 & 0 & {\left[\Pi_{34 l j f}^{S}\right.}
\end{array}\right]^{\mathrm{T}}\right] .
\end{aligned}
$$

By applying Young's inequality and the Schur complement, then further considering the quadratic form of matrix $\widehat{Y}$, (A.8) is guaranteed by (23) with additional dealing with the non-negativity of double convex summations by applying "Proposition 2" in [26].

\section{REFERENCES}

[1] G. C. Goodwin, H. Kong, G. Mirzaeva, and M. M. Seron, "Robust model predictive control: reflections and opportunities," Journal of Control and Decision, vol. 1, no. 2, pp. 115-148, May 2014

[2] D. Q. Mayne, "Model predictive control:Recent developments and future promise," Automatica, vol. 50, no. 12, pp. 2967-2986, December 2014.

[3] M. B. Saltık, L. Özkan, J. H. Ludlage, S. Weiland, and P. M. Van den Hof, "An outlook on robust model predictive control algorithms: Reflections on performance and computational aspects," Journal of Process Control, vol. 61, pp. 77-102, January 2018.

[4] B. Ding, "Constrained robust model predictive control via parameter-dependent dynamic output feedback," Automatica, vol. 46, no. 9, pp. 1517-1523, September 2010.

[5] D. Famularo and G. Franzè, "Output feedback model predictive control of uncertain norm-bounded linear systems," International Journal of Robust and Nonlinear Control, vol. 21, no. 8, pp. 838-862, May 2011.

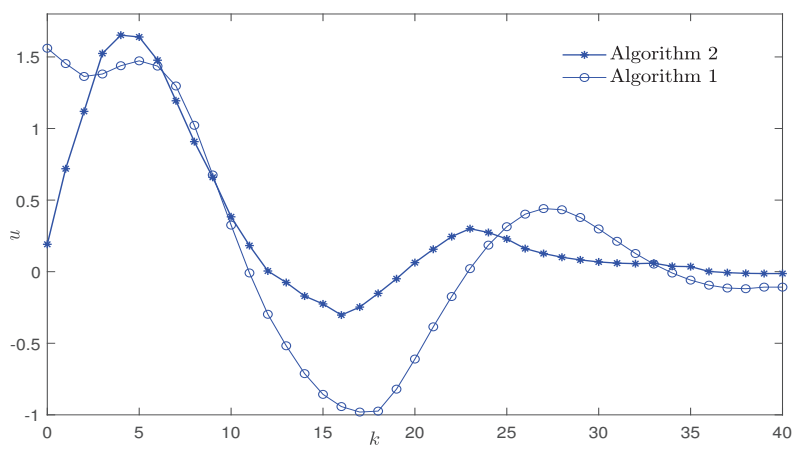

Fig. 5. The comparison on control inputs, Example 1.

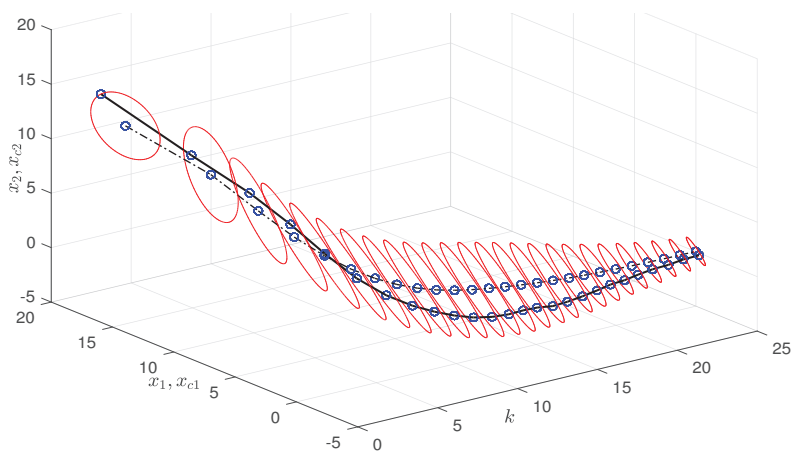

Fig. 6. The responses of $x_{c}(k), x(k)$ and $e(k)$, Algorithm 1, Example 2

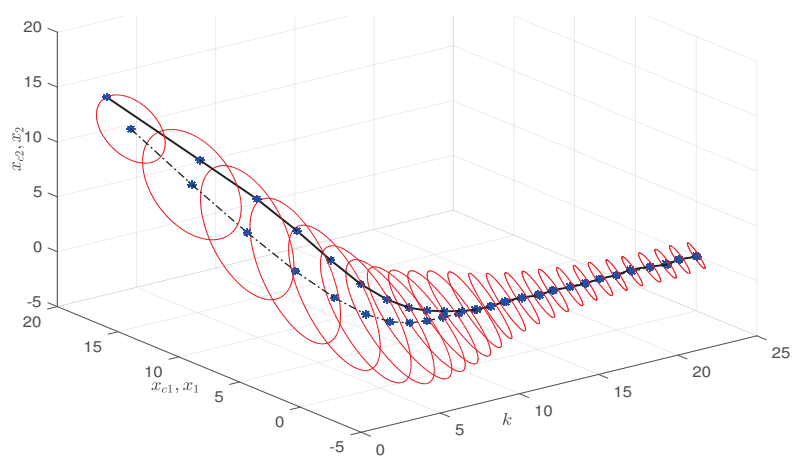

Fig. 7. The responses of $x_{c}(k), x(k)$ and $e(k)$, Algorithm 2, Example 2.

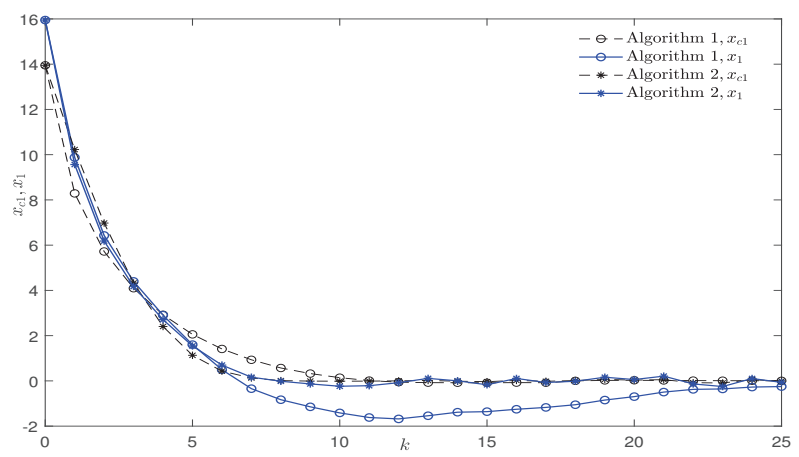

Fig. 8. The responses of $x_{c 1}, x_{1}$ for the compared Algorithms, Example 2. 


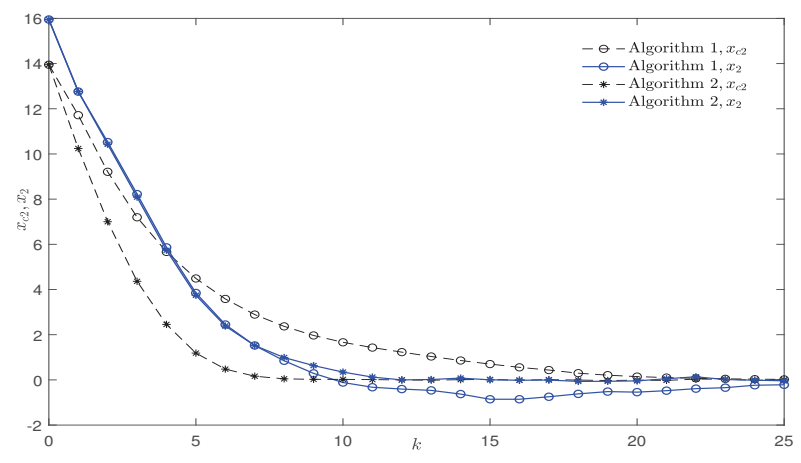

Fig. 9. The responses of $x_{c 2}, x_{2}$ for the compared Algorithms, Example 2.

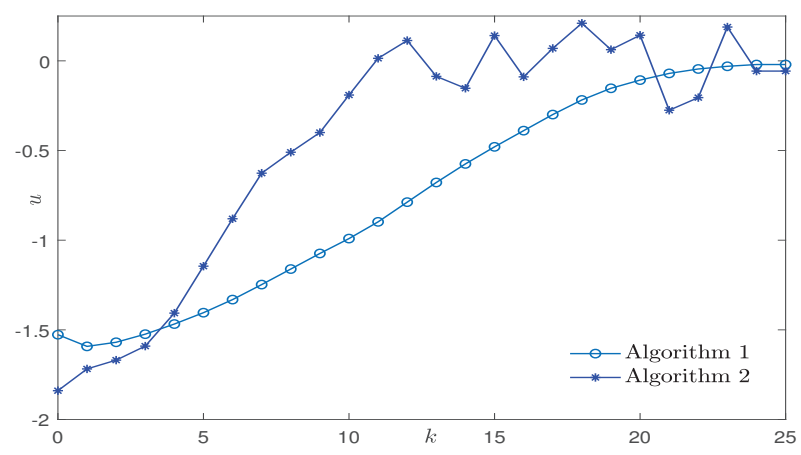

Fig. 10. The comparison on control inputs, Example 2.

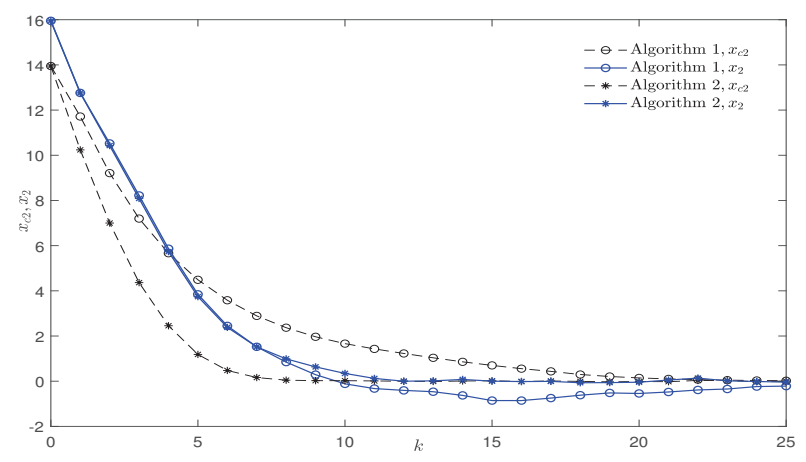

Fig. 11. The responses of $x_{c 2}, x_{2}$ for the compared Algorithms, Example 2.

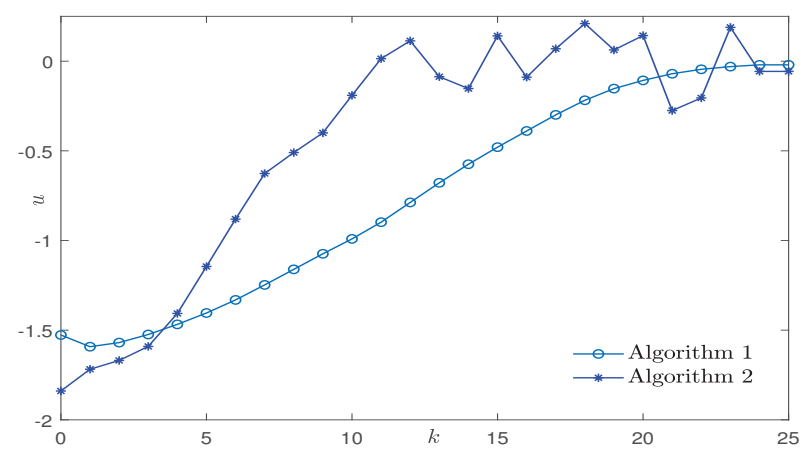

Fig. 12. The comparison on control inputs, Example 2.
[6] W. Yang, J. Gao, G. Feng, and T. Zhang, "An optimal approach to output-feedback robust model predictive control of LPV systems with disturbances," vol. 26, no. 5, pp. 3253-3273, January 2016.

[7] S. Subramanian, S. Lucia, and S. Engell, "A novel tubebased output feedback MPC for constrained linear systems," American Control Conference (ACC), pp. 30603065, May 2017.

[8] D. Q. Mayne, Q. David, S. V. Raković, R. Findeisen, and F. Allgöwer, "Robust output feedback model predictive control of constrained linear systems," Automatica, vol. 42, no. 7, pp. 1217-1222, May 2006.

[9] D. Q. Mayne, S. V. Raković, R. Findeisen, and F. Allgöwer, "Robust output feedback model predictive control of constrained linear systems: Time varying case," Automatica, vol. 45, no. 9, pp. 2082-2087, September 2009.

[10] X. Ping, "Output feedback robust MPC based on off-line observer for LPV systems via quadratic boundedness," Asian Journal of control, vol. 19, no. 4, pp. 1641-1653, July 2017.

[11] B. Ding, H. Pan, "Output feedback robust MPC with one free control move for linear polytopic uncertain system with bounded disturbance," Automatica, vol. 50, no. 11, pp. 2929-2935, November 2014.

[12] B. Ding, and H. Pan, "Output feedback robust MPC for LPV system with polytopic model parametric uncertainty and bounded disturbance," International Journal of Control, vol. 89, no. 8, pp. 1554-1571, 2016.

[13] B. Ding, C. Gao, and X. Ping, "Dynamic output feedback robust MPC using general polyhedral state bounds for the polytopic uncertain system with bounded disturbance," Asian Journal of Control, vol. 18, no. 2, pp. 699-708, January 2016.

[14] X. Ping, and B. Ding, "Off-line approach to dynamic output feedback robust model predictive control," Systems \& Control Letters, vol. 62, no. 11, pp. 1038-1048, November 2013.

[15] J. R. Colombo Junior, R. K. H. Galvão, and E. Assuncão, "A new OFRMPC formulation with on-line synthesis of the dynamic output feedback controller," International Journal of Robust and Nonlinear Control, vol. 27, no. 17, pp. 3921-3936, November 2017.

[16] T. Shi, Z. G. Wu, and H. Su, "Improved dynamic output feedback RMPC for linear uncertain systems with input constraints," International Journal of Robust and Nonlinear Control, vol. 26, no. 12, pp. 2729-2742, January 2016.

[17] B. Ding, X. Ping and H. Pan, "On Dynamic Output Feedback Robust MPC for Constrained Quasi-LPV Systems," International Journal of Control, vol. 86, no. 12, pp. 22152227, June 2013.

[18] D. Li, Y. Xi, "Synthesis of dynamic output feedback RMPC with saturated inputs," Automatica, vol. 49, no. 4, pp. 949-954, April 2013.

[19] O. Toker and H. Ozbay, "On the NP-hardness of solving bilinear matrix inequalities and simultaneous stabilization with static output feedback," American Control Conference, vol. 4, pp.2525-2526, July 1995. 
[20] M. S. Sadabadi, D. Peaucelle, "From static output feedback to structured robust static output feedback: A survey," Annual reviews in control, vol. 42, no. 3, pp. 11-26, September 2016.

[21] L. El Ghaoui, F. Oustry and M. AitRami, "A cone complementarity linearization algorithm for static outputfeedback and related problems," IEEE Transactions on Automatic Control, vol. 42, no. 8, pp. 1171-1176, September 1997.

[22] S. Boyd, L. El Ghaoui, E. Feron, and V. Balakrishnan, "Linear matrix inequalities in system and control theory," Siam, 1994.

[23] H. Li and M. Fu, "A linear matrix inequality approach to robust $H_{\infty}$ filtering," IEEE Transactions on Signal Processing, vol. 45, no. 9, pp. 2338-2350, September 1997.

[24] H. Kheloufi, F. Bedouhene, A. Zemouche, A. Alessandri, "Observer-based stabilisation of linear systems with parameter uncertainties by using enhanced LMI conditions," International Journal of Control, vol. 88, no. 6, pp. 11891200, January 2015

[25] K. Derinkuyu, M. C. Pinar, "On the S-procedure and some variants," Mathematical Methods of Operations Research, vol. 64, no. 1, pp. 55-77, August 2006.

[26] A. Sala and C. Ariño, "Asymptotically necessary and sufficient conditions for stability and performance in fuzzy control: Application of Polya's theorem," Fuzzy Sets and Systems, vol. 158, no. 24, pp. 2671-2686, December 2007.

[27] P. Gahinet, A. Nemirovski, A. J. Laub, and M. Chilali, LMI control toolbox for use with matlab, User's guide, The Math Works Inc., Natick, MA, USA, 1995.

[28] H. Li, Y. Pan, P. Shi, "Switched fuzzy output feedback control and its application to mass-spring-damping system," IEEE Transactions on Fuzzy Systems, vol. 24, no. 6, pp. 1259-1269, 2016.

[29] K. Tanaka, H. O. Wang, "Fuzzy control systems design and analysis: a linear matrix inequality approach", John Wiley \& Sons, 2004.

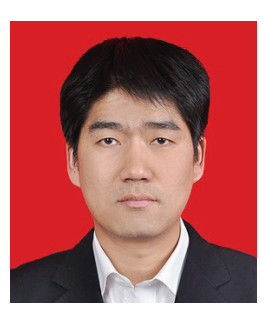

Xubin Ping received the Bachelor's degree from Northwest University, Xi'an, China, in 2005 and the Master's degree from the East China University of Science and Technology, Shanghai, China, in 2008 and the Ph.D degree from Xi' an Jiaotong University, Xi'an, China, in 2013. His research interests include robust control, model predictive control.

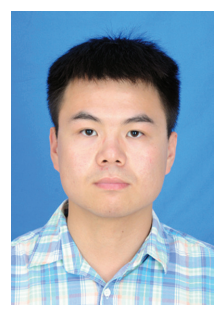

Sen Yang received the Bachelor's degree from Xi' an University of Science and Technology, Xi' an, China, in 2018. He is currently Master student major in control science and engineering with Xidian University, Xi'an, China. His research interests cover model predictive control and its applications.

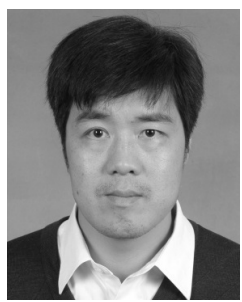

Baocang Ding was born in Hebei Province, China. He received the M.S. degree from the China University of Petroleum, Beijing, China, in 2000 and the $\mathrm{Ph}$.D. degree from Shanghai Jiaotong University, Shanghai, China, in 2003. From September 2005 to September 2006, he was a Postdoctoral Research Fellow in Department of Chemical and Materials Engineering, University of Alberta, Canada. From November 2006 to August 2007, he was a Research Fellow in the School of Electrical and Electronic Engineering, Nanyang Technological University, Singapore. From September 2003 to August 2007, he was an Associate Professor in Hebei University of Technology. From September 2007 to December 2008, he was a Professor in Chongqing University. From January 2009 to September 2019, he was a Professor in Xi'an Jiaotong University. He is currently a Professor with Chongqing University of Posts and Telecommunications, Chongqing, China. His research interests include predictive control, fuzzy control, networked control, and distributed control systems.

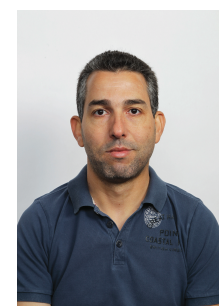

Tarek Raiissi received the Engineering degree from National Engineering School of Tunis in 2000, the Master degree in Automatic Control from Central School of Lille in 2001, the Ph.D. from the University of Paris XII in 2004 and the Accreditation to Supervise/Conduct Research (HDR) from the University of Bordeaux in 2012. From 2005 to $2011 \mathrm{He}$ was an Associate Professor at the University of Bordeaux. Currently, He is a Full Professor at the Conservatoire National des Arts et Metiers, Paris, France. He is a member of the IFAC Technical Committee "Modeling, Identification and Signal Processing" and a Senior member of IEEE. His research interests include fault detection and isolation, nonlinear systems estimation and robust control.

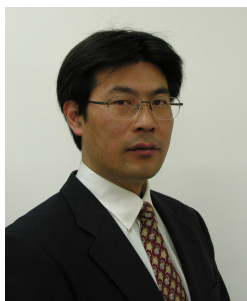

Zhiwu Li received the B.S. degree in mechanical engineering, the M.S. degree in automatic control, and the Ph.D. degree in manufacturing engineering from Xidian University, Xi'an, China, in 1989, 1992, and 1995, respectively, where he joined in 1992. He is also with the Institute of Systems Engineering, Macau University of Science and Technology, Taipa, Macau, China. He is listed in Who's Who in the World (27th Ed., Marquis, 2010). His current research interests include Petri net theory and application, supervisory control of discrete event systems, workflow modeling and analysis, system reconfiguration, game theory, and data and process mining. He is a Fellow of IEEE. He is the Founding Chair of the Xi' an Chapter of the IEEE Systems, Man, and Cybernetics Society. 